\title{
Establishing Best Practices for X-57 Maxwell CFD Database Generation
}

\author{
Jared C. Duensing* \\ Science and Technology Corporation, M/S 258-2, Moffett Field, California, 94035 \\ Seung Y. Yoo ${ }^{\dagger}$ \\ NASA Armstrong Research Center, 4800 Lilly Ave, Edwards, California, 93523 \\ Daniel Maldonado \\ Science and Technology Corporation, M/S 258-2, Moffett Field, California, 94035 \\ Jeffrey A. Housman ${ }^{\S}$, James C. Jensen ${ }^{\mathbb{I}}$ and Cetin C. Kiris" \\ NASA Ames Research Center, M/S 258-2, Moffett Field, California, 94035
}

\begin{abstract}
The X-57 Maxwell is NASA's latest electric airplane concept that has been simulated for aerodynamic performance using the structured overset and unstructured grid solvers within the Launch Ascent and Vehicle Aerodynamics (LAVA) solver framework as well as the unstructured polyhedral grid solver in Star-CCM+ for code-to-code comparison. In order to validate the predictions, comparisons were made between the CFD solutions and experimental data collected in the 12-foot Low-Speed Wind Tunnel at NASA Langley Research Center. The simulations are in preparation for the development of a comprehensive aerodynamic database which will assess aircraft performance at a variety of conditions. The findings from these simulations will establish the best practices for mesh resolution, numerical discretization, and turbulence modeling to be used for this database. Preliminary database results have shown that best-practices learned from the initial validation simulations will potentially reduce error in X-57 aerodynamic loads and moments relative to experiment by up to $14 \%$.
\end{abstract}

\section{Nomenclature}

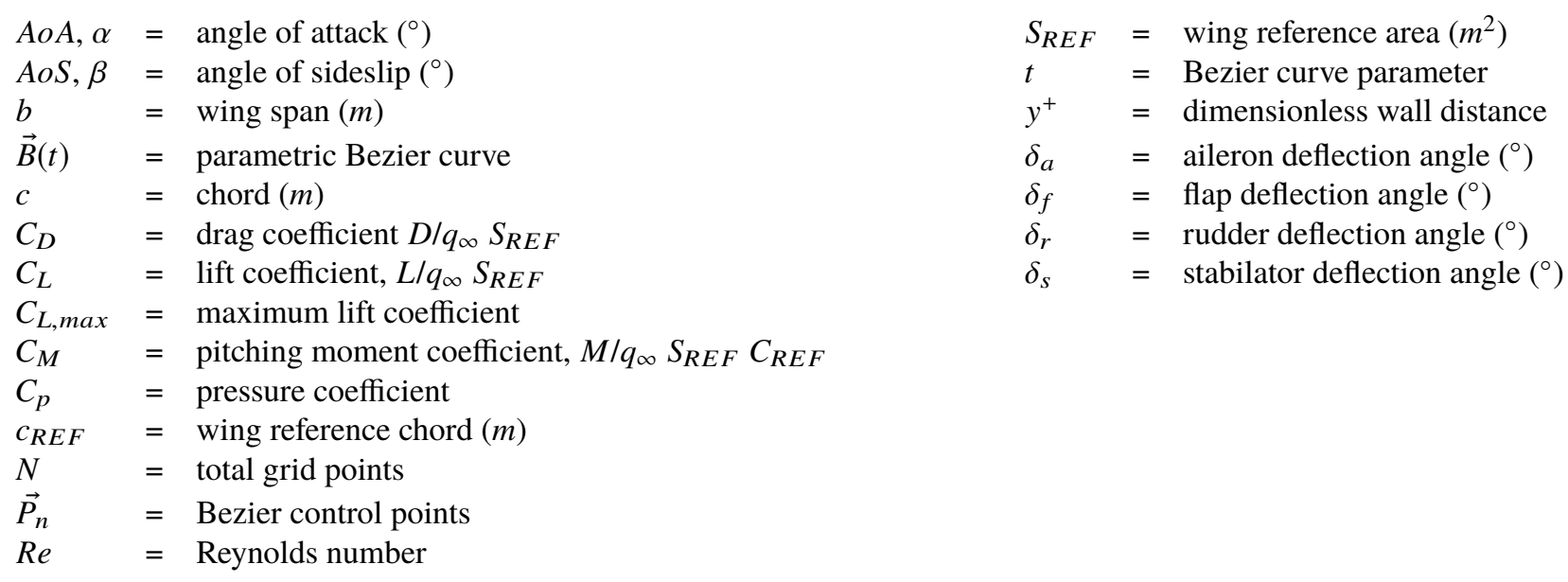

\footnotetext{
*Computational Aerosciences Branch, NAS Division, Moffett Field, California, 94035, jared.c.duensing @nasa.gov

†Controls and Dynamics Branch, P.O. Box 273/MS 4840D, Edwards, California, 93523, seung.y.yoo@ nasa.gov

†Computational Aerosciences Branch, NAS Division, Moffett Field, California, 94035, daniel.maldonado@nasa.gov

${ }^{\S}$ Computational Aerosciences Branch, NAS Division, Moffett Field, California, 94035, AIAA Senior Member, jeffrey.a.housman@ nasa.gov

IIComputational Aerosciences Branch, NAS Division, Moffett Field, California, 94035, AIAA Member, james.c.jensen@nasa.gov

"Computational Aerosciences Branch, NAS Division, Moffett Field, California, 94035, AIAA Senior Member, cetin.c.kiris@ nasa.gov
} 


\section{Introduction}

$\mathbf{I}^{\mathrm{N}}$ recent years, the National Aeronautics and Space Administration (NASA) has been actively participating in the Iresearch and development of electrically-powered aircraft. The latest X-plane concept known as the X-57 Maxwell [1] is NASA's solution to this problem and researchers have been working to demonstrate the feasibility of this aircraft. With its high aspect ratio wing design, stability controls, and twelve high-lift propulsors used for distributed propulsion, simulating the governing physics of this aircraft requires engineering tools that can handle high levels of geometric and physical complexity. The Launch Ascent and Vehicle Aerodynamics (LAVA) solver framework [2] developed at NASA Ames Research Center is one such tool researchers are using to study the X-57 vehicle. This work is being done in collaboration with NASA Armstrong Research Center, where CFD analysis is done using the commercial software package Star-CCM+ [3]. The aerodynamic database constructed through this collaboration will be crucial in developing a safe flight test program for the electric vehicle.

While the final objective of this study is to develop an aerodynamic database for X-57 Modification (Mod.) IV, other X-57 concepts known as Mod. II and III are being analyzed in preparation for this goal. The Mod. II design has a lower aspect ratio wing design with only two electrically powered propulsors while the Mod. III is identical to the Mod. IV, but excludes the high-lift propulsors. The majority of work presented here has been for the unpowered Mod. III wind tunnel model for a variety of conditions. Figure 1 depicts a rendering of this aircraft design and a schematic illustrating the control surface deflections that will be simulated. These preliminary CFD simulations for the Mod. III geometry will be discussed at length, including grid sensitivity of the solution, impacts of solver settings, and impacts of simulating the aircraft in free air versus wind tunnel. Experimental data collected in the 12-foot Low Speed Wind Tunnel (LST) at NASA Langley Research Center will be used to validate the results. The lessons learned from these initial simulations will establish best practices for future CFD analysis on this aircraft.

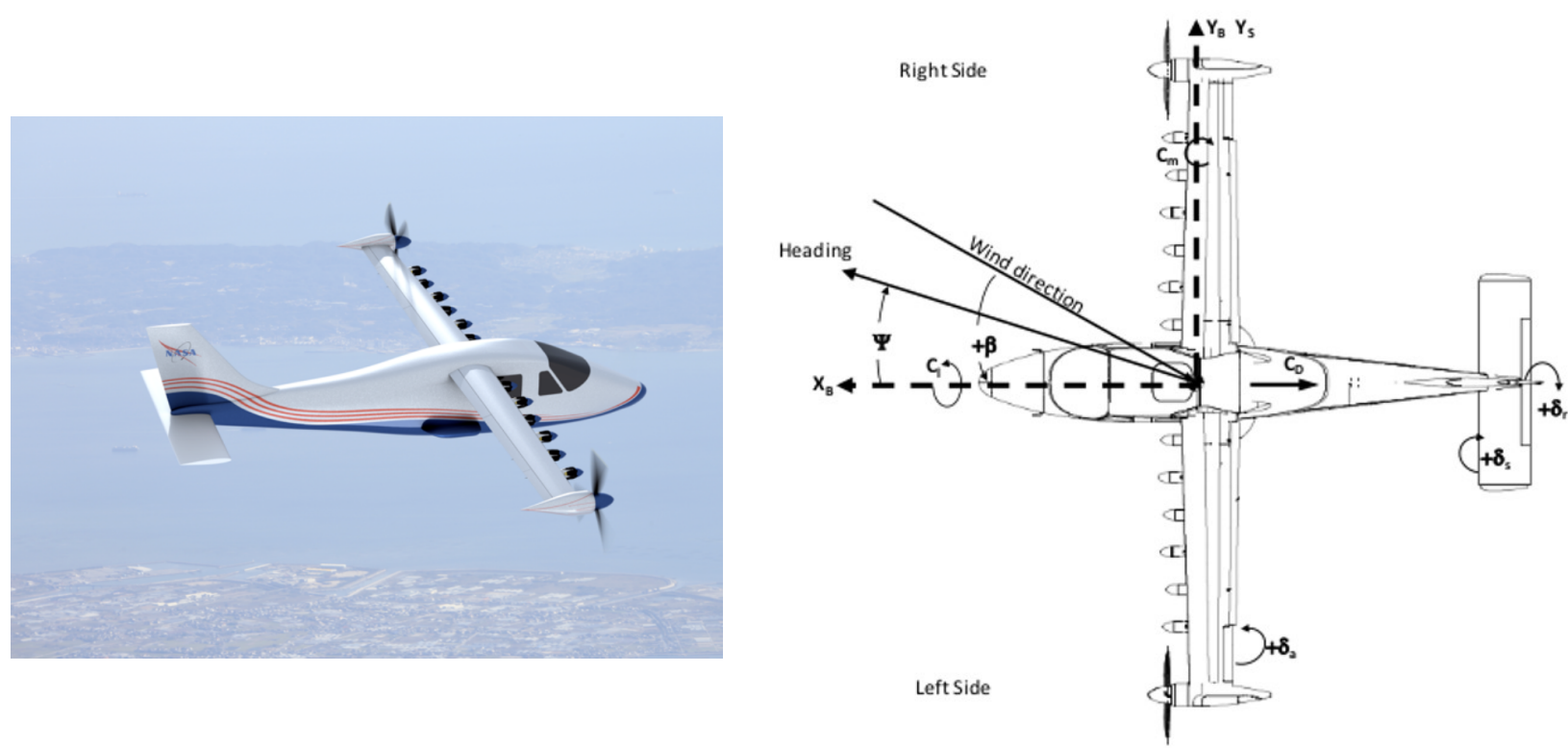

(a) 3-D rendering [4].

(b) Top-view schematic.

Fig. 1 X-57 Maxwell Mod. III Aircraft.

\section{Wind Tunnel Experiment}

Experimental data for the X-57 used for code validation was collected in the 12-foot Low-Speed Tunnel at NASA Langley Research Center [5]. This tunnel is an open circuit tunnel placed inside of a $60-\mathrm{ft}$ diameter spherical casing and operated at atmospheric pressure. The test section has regular octagonal shape measuring 12 feet in width and height and a total length of 15 feet. A fan downstream of the test article draws air through the test section, which then recirculates 
through the spherical chamber and reenters through the honeycomb screens placed at the inlet shown in Fig. 2.

The X-57 model is mounted on a sting and C-strut system that is used for static force and moment testing. This system allows any angle of attack to be tested between -10 and $90^{\circ}$ by translating the sting along the C-strut, while the strut can also be rotated about a vertical axis to create any desired sideslip angle. Data acquisition is performed on a UNIX based computer system which processes static force and moment measurements taken from an internally placed strain-gage balance. These forces and moments are then nondimensionalized using reference dimensions and conditions to yield the coefficients that will be used to validate any subsequent CFD simulations.

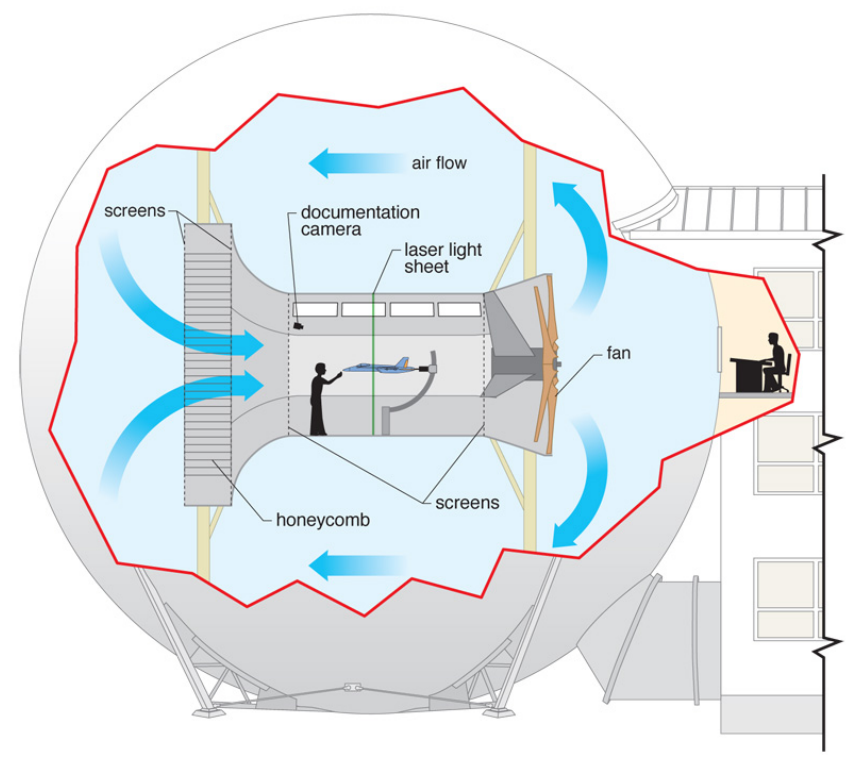

(a) Operational schematic of the 12-foot Low-Speed Tunnel at NASA Langley Research Center.

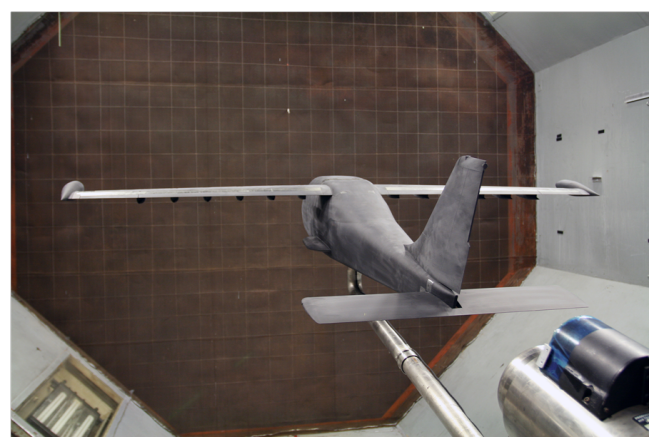

(b) Rear view of the installed X-57 test article. Image courtesy of Gerald Lee Pollard, NASA Langley.

Fig. 2 X-57 Maxwell Mod. III experimental set-up.

\section{Computational Methodologies}

The LAVA solver framework is one of the CFD software packages being utilized to simulate X-57 performance. This framework provides the user with a variety of mesh paradigms which are chosen based on application and user necessity. The supported mesh types include structured curvilinear, structured Cartesian, and unstructured arbitrary polyhedral. For this particular application, the structured curvilinear and unstructured paradigms were chosen for the analysis. The structured curvilinear option requires the generation of structured overlapping surface grids that are hyperbolically grown into near-body volume grids, which provide the benefit of a resolved boundary layer, the ability to easily deflect control surfaces, and rapid volume grid generation. The unstructured option requires the generation of a surface triangulation grown into arbitrary polyhedral cells, which provides the benefits of a resolved boundary 
layer and quick surface mesh generation, but a new mesh must be generated for each control surface deflection angle. Subsection IV.A will detail the advantages of the structured overset topology with respect to the quick regeneration of surface and volume meshes.

The compressible Reynolds Averaged Navier-Stokes (RANS) equations are solved on the structured curvilinear and unstructured grids. Several conservative finite-difference formulations are available within the framework for the structured curvilinear option, while a second-order, finite-volume formulation is supported in the unstructured option. Multiple turbulence modeling approaches are available, including the Spalart-Allmaras [6] (SA) model which will be utilized for this study. The LAVA capabilities utilized for this study using both the unstructured and structured overset paradigms will be discussed in section IV.

STAR-CCM+ is a commercial CFD software package consisting of CAD/geometry manipulation tools, grid generator, flow solver, and post processing tools. The framework allows the users to perform the analysis from beginning to the end within STAR-CCM+. The grid generator is robust and capable of creating grids of various topologies, including polyhedral, tetrahedral, and cartesian. Any of the mentioned topologies can be combined with a prism layer mesher to adequately capture the boundary layer. A polyhedral grid topology would eventually be the type selected for this study. The CAD/geometry manipulation tools were used to model the control surface deflection, streamlining the process of simulating aircraft of various control surface deflections.

STAR-CCM+ solves Reynolds-Averaged Navier-Stokes equations in the cell-centered, finite-volume formulation. Two separate schemes are available to discretize the inviscid flux terms: Roe flux-difference splitting scheme and ASUM+ flux-vector splitting scheme [7] [8]. Hybrid Gauss Least Square method was used to estimate the gradients. To limit the estimated gradient, several limiters are available: Venkatakrishnan [9], Min-mod, and modified Venkatakrishnan. Multiple turbulence models are available including the Spalart-Allmaras model and Shear-Stress Transport model. Section V details the models and settings utilized for the Star-CCM+ simulations.

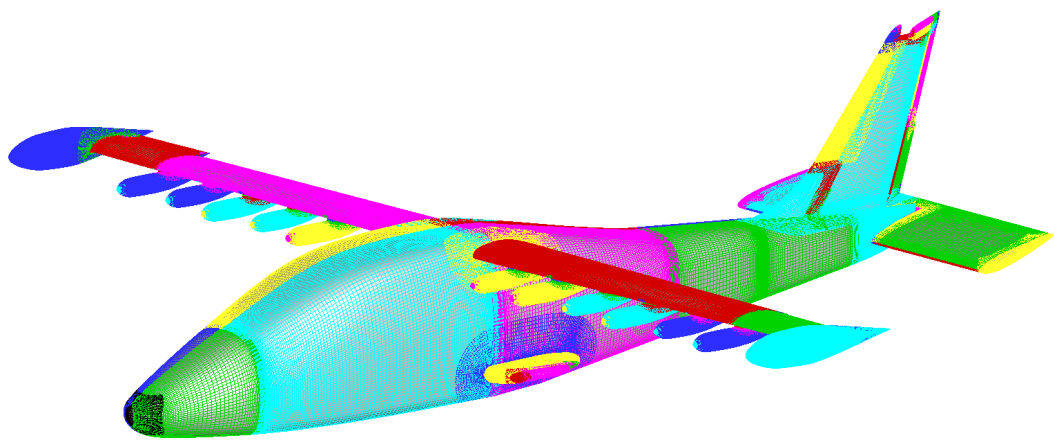

Fig. 3 Structured overset surface grids for coarse level mesh.

\section{Geometry Preparation and Grid Generation}

Structured overset grids have been generated for all studied aircraft configurations, which include all relevant deflections of the aileron, flap, rudder, and stabilator. Geometry was provided as a Standard Exchange of Product (STEP) file for the nominal (undeflected) geometry. Table 1 summarizes the reference quantities used for non-dimensionalizations of aerodynamic variables in the Mod. III wind tunnel scale geometry. It should be noted that spacings, stretching ratios and grid point counts for LAVA Curvilinear, LAVA Unstructured and Star-CCM+ were all independently chosen, but all follow best practices outlined in the HLPW3 gridding guidelines.

\section{A. LAVA Overset Structured Grid Generation}

Geometry modification was required to make overset structured mesh generation possible while also allowing all control surfaces to be articulated within the grid script. This step was conducted within the ANSA program [10], a $\mathrm{CAD}$ and mesh generating software used for geometry modifications and basic mesh construction. Artificial gaps were created between all rotating and stationary components such that structured grid patches could be generated for any desired movement. A surface triangulation of the final geometry was then exported as a StereoLithography (STL) file to be used as the projection surface for any subsequent grids. Additionally, structured surface grid patches were 
Table 1 X-57 Mod. III Reference Quantities for Wind Tunnel Scale Model

\begin{tabular}{|l|c|}
\hline Quantity & Value \\
\hline Mean Aerodynamic Chord, $c_{R E F}$ & $0.1234 \mathrm{~m}$ \\
Wing Semi-Span, $b$ & $1.8314 \mathrm{~m}$ \\
Wing Reference Area, $S_{R E F}$ & $0.2236 \mathrm{~m}^{2}$ \\
Mach Number & 0.052 \\
Moment Reference Center, $M R C$ & $(0.7442,0.0,0.3042) \mathrm{m}$ \\
Reynolds Number Based on MAC, $R e$ & 121,600 \\
Reference Static Pressure, $P_{R E F}$ & $101,325 \mathrm{~Pa}$ \\
Reference Static Temperature, $T_{R E F}$ & $288.1 \mathrm{~K}$ \\
\hline
\end{tabular}

placed on the geometry and exported in Plot3D (P3D) format. These structured grid patches were then imported into Pointwise [11] to be projected onto the reference geometry, represented by the CAD. Appropriate grid spacings, point distributions and stretching ratios were then assigned to each structured patch using guidelines established by the 3rd AIAA High Lift Prediction Workshop [12] (HLPW-3) to create the coarse level mesh for the grid refinement study. These guidelines recommend a maximum stretching ratio of 1.25 , chordwise leading edge and trailing edge spacing of $0.1 \%$ of local chord, and 5 points on every finite-thickness trailing edge which yielded the grid in Fig. 3 .

Once refined, these structured patches were then exported into P3D format and imported into Chimera Grid Tools [13] (CGT) to create the overlap between adjacent patches and create additional collar and cap grids [14]. For some grids, a gap was intentionally created between moving components and the fixed geometry to allow control surfaces to deflect as desired. This procedure was used for the ailerons, flap, stabilator and rudder. Once these components were rotated to a desired angle, quadratic Bezier curves [15] given by Eq. 1 were used to connect the moving and stationary components so that any discontinuities and sharp transitions between rotating and stationary components were eliminated (i.e., between wing and aileron),

$$
\vec{B}(t)=(1-t)^{2} \vec{P}_{0}+2(1-t) t \vec{P}_{1}+t^{2} \vec{P}_{2}, 0 \leq t \leq 1
$$

where $t$ is the curve parameter, and $\vec{P}_{0}, \vec{P}_{1}$, and $\vec{P}_{2}$ are the control points. The curves were then patched into a surface using transfinite interpolation (TFI) to form the final control surface patch. Figure 4 shows the Bezier curves that define the transition from the wing to aileron surface, and Fig. 5 shows the TFI patch in pink constructed from these curves and then overlapped with surrounding grids. This methodology provided a realistic representation of the geometry for any configuration while also permitting any deflection. It is anticipated that this advantage of structured overset meshes will greatly reduce the grid generation overhead during the final database generation.

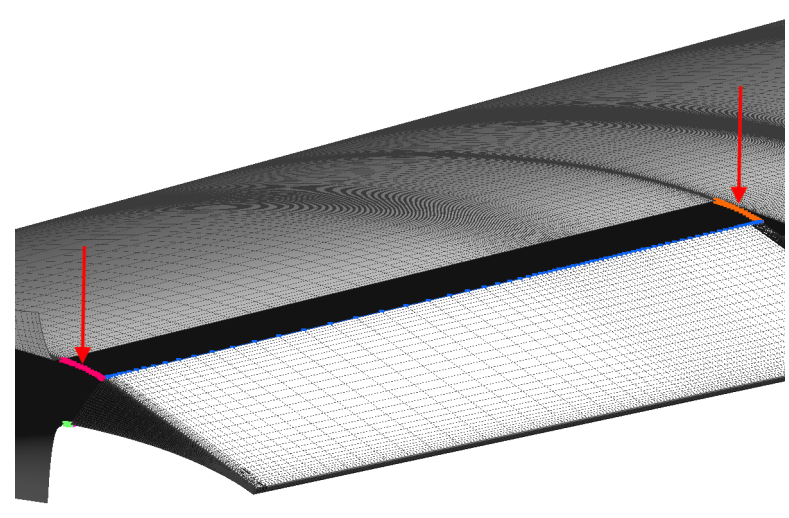

(a) $\delta_{a}=\mathbf{+ 1 0}$

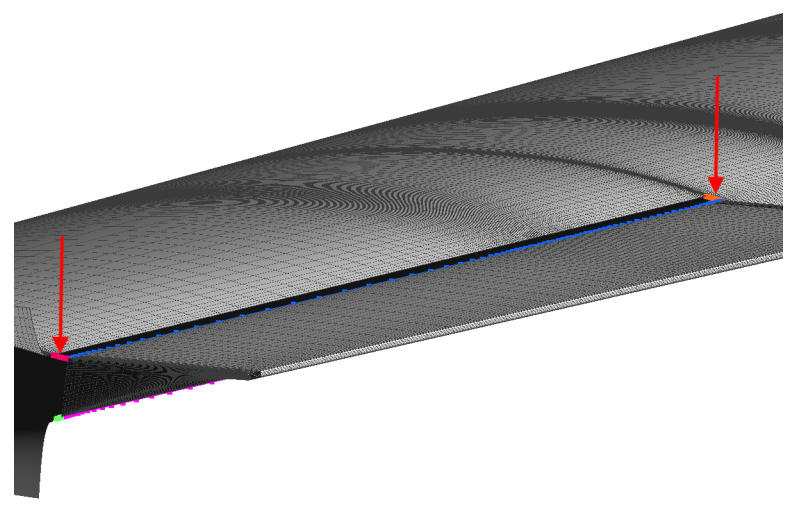

(b) $\delta_{a}=\mathbf{- 1 0}{ }^{\circ}$

Fig. 4 Floating aileron grids for selected deflections with computed Bezier curves (colored). 


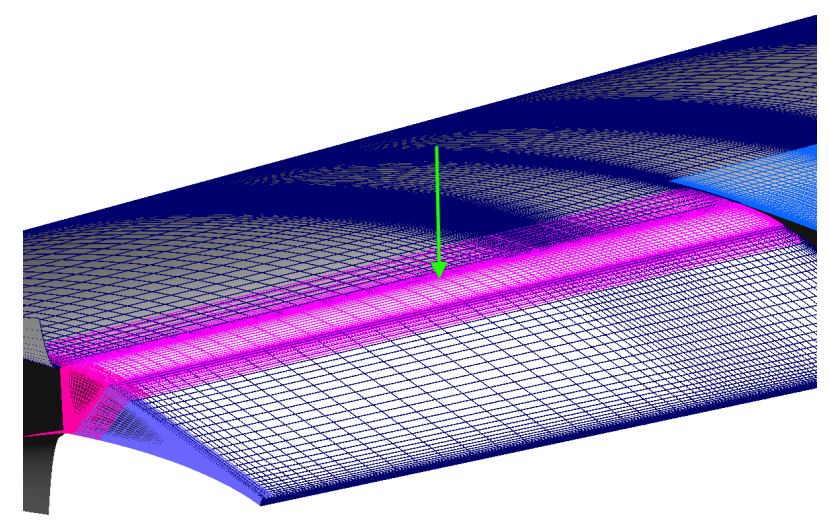

(a) $\delta_{a}=\mathbf{+ 1 0}$

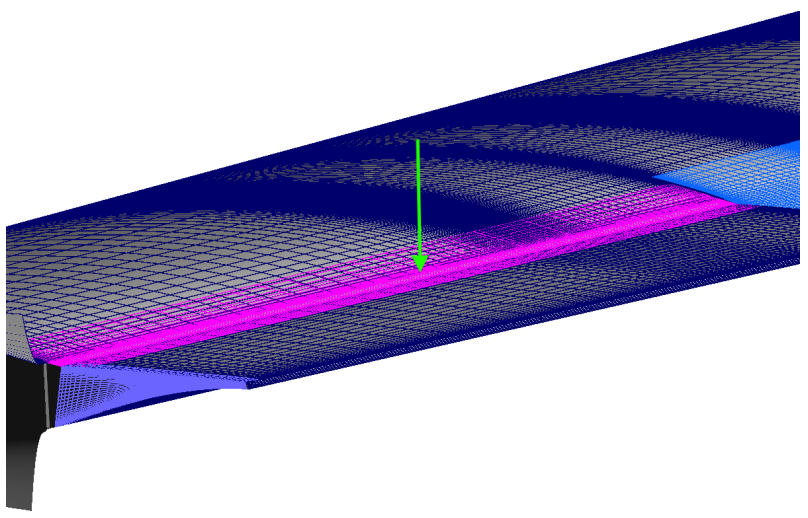

(b) $\delta_{a}=\mathbf{- 1 0}$

Fig. 5 Patched aileron grid for selected deflections using transfinite interpolation (TFI) surfaces and hyperbolic marching to overlap with surrounding grids (shown in pink).

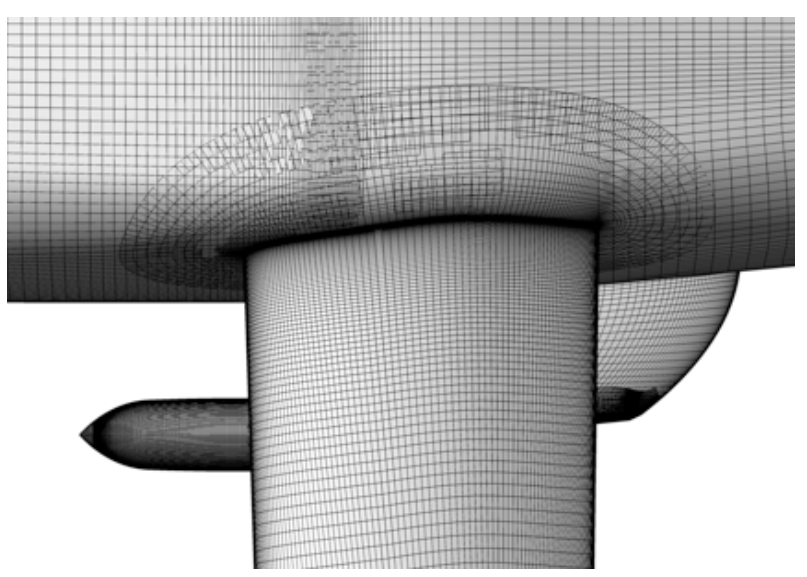

(a) Coarse: 80 million points

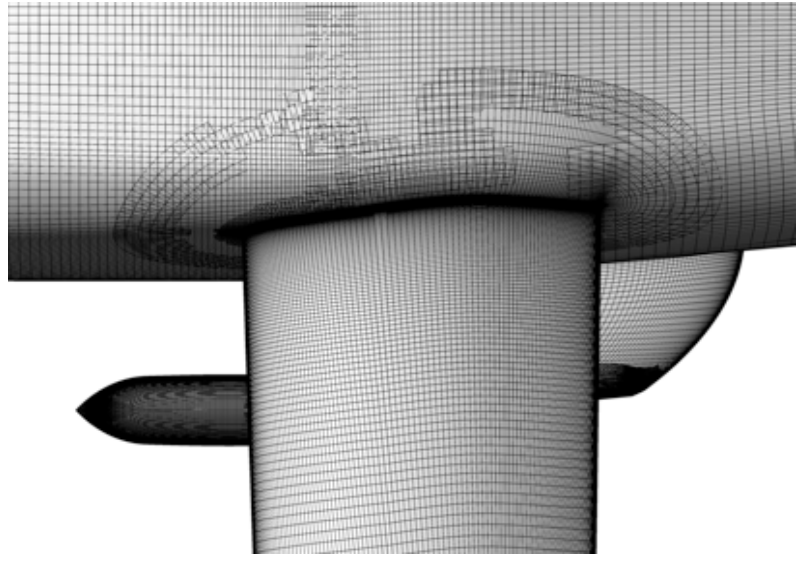

(b) Medium: 159 million points

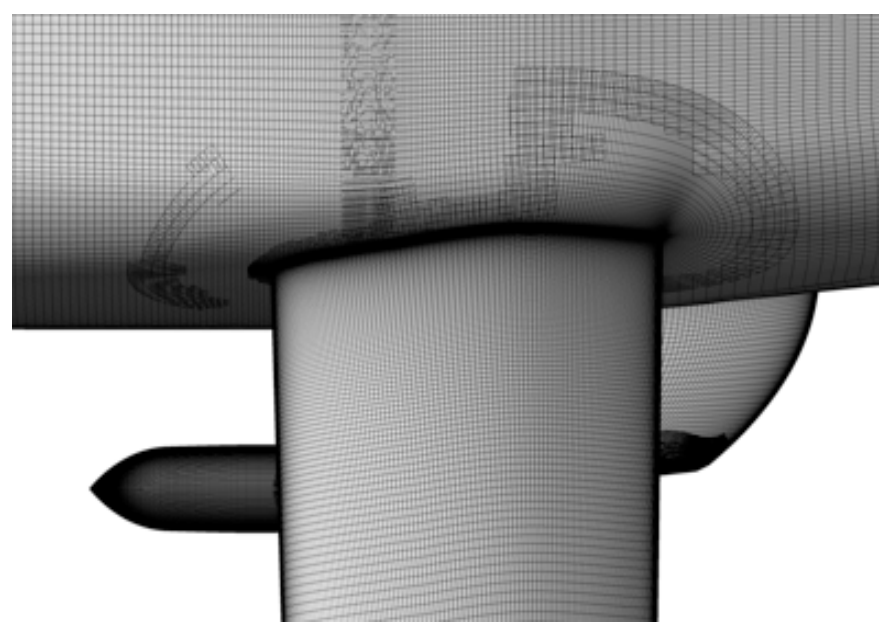

(c) Fine: 322 million points

Fig. 6 Overset mesh refinement surface grids. 
The first structured overset grid was constructed as the coarse level grid for the initial mesh refinement study. In this case, $\alpha=6.0^{\circ}, \beta=5.0^{\circ}, \delta_{a}=10.0^{\circ}, \delta_{r}=15.0^{\circ}$, and $\delta_{s}=7.5^{\circ}$, were selected to mimic an off-design flight condition. Volume meshes were then hyperbolically grown from the surfaces with $y^{+} \approx 1$ computed from free stream conditions given in Table 1 to adequately resolve the boundary layer and grown with a stretching ratio of 1.2. Cartesian box grids were then constructed around every major aircraft component with a grid spacing commensurate to the outer boundary spacing of the body-fitted volume grids. A final Cartesian box grid was constructed around all volumes to extend the far-field to 100 fuselage lengths away from the aircraft body. This resulted in a total of 80 million grid points for the coarse level grid, with 273 individual overset meshes. A consistent mesh refinement was then applied to the coarse grid to create the medium and fine level meshes with updated mesh parameters given in the HLPW-3. This resulted in a mesh refinement ratio of approximately 2.0 for the total grid points between each grid level, or 159 million grid points for the medium grid and 322 million grid points for the fine grid, shown in Fig. 6. For this particular grid study, the $y^{+}$ wall spacing and stretching ratios were also reduced in the wall normal direction to ensure additional resolution in the boundary layer as the mesh is refined. Specifically, the medium and fine grid levels utilized a $y^{+} \approx 0.67$ and $y^{+} \approx 0.44$ respectively, while all stretching ratios were reduced to 1.16 and 1.12 respectively.

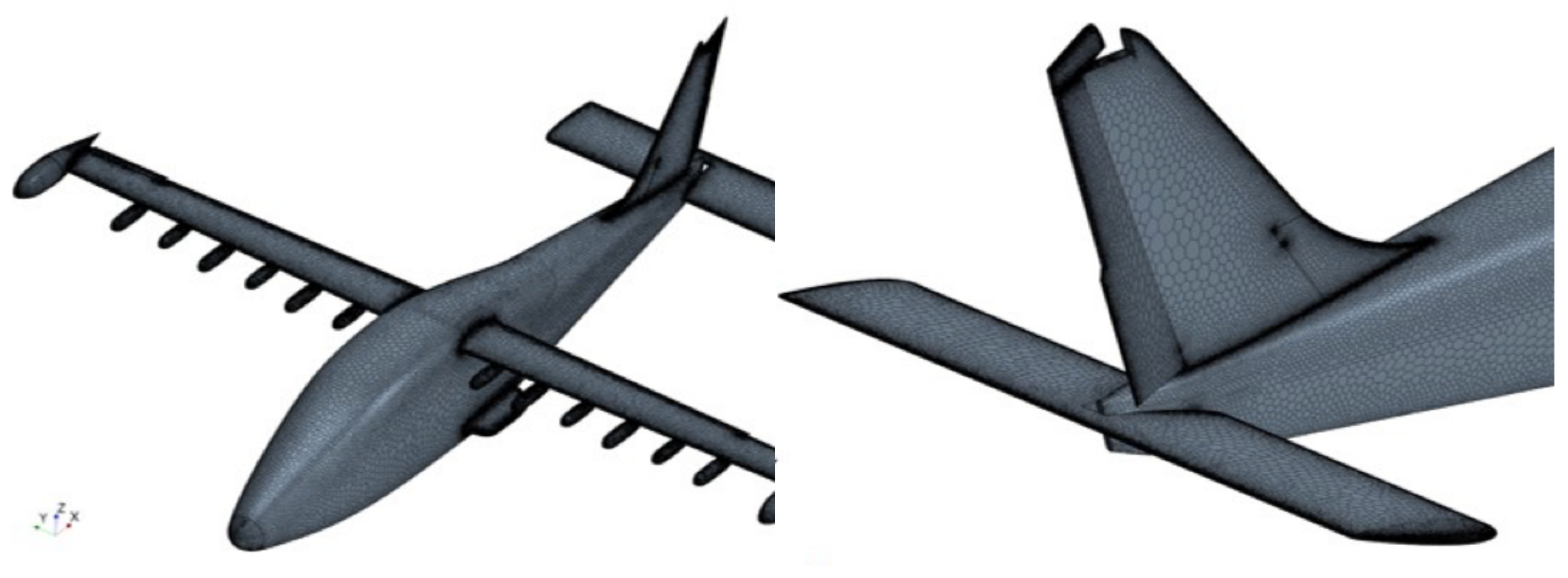

(a) Full Isometric View

(b) Empennage

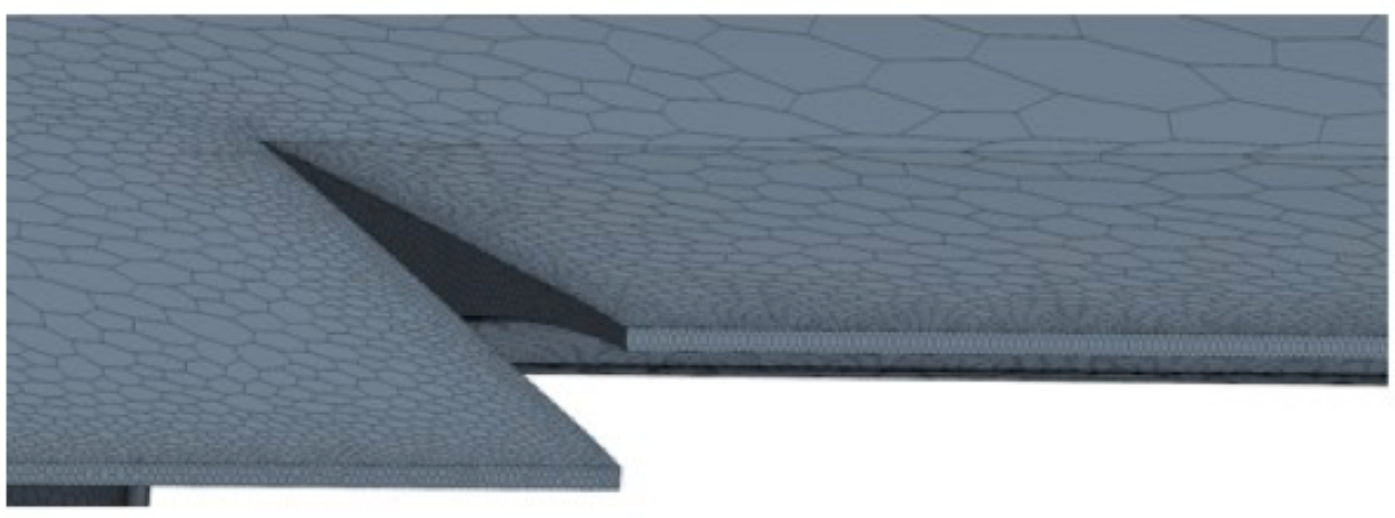

(c) Deflected Aileron

Fig. 7 Surface polyhedral cells for the Star-CCM+ unstructured grid.

\section{B. LAVA Unstructured Grid Generation}

For unstructured grid simulations, geometry preparation to generate grids is similar to the structured option. However, fewer modifications to the geometry are required. Using the ANSA program, the entirety of the surface mesh triangulation could be generated using the same spacing and stretching guidelines established by the HLPW-3. The 
resulting triangulation was then exported as an STL file and imported into the StarCCM program to generate the volume grid. In order to resolve the boundary layer in a manner consistent with the structured overset grid, prismatic cells were grown near the body and transitioned to arbitrary polyhedral cells that extend to the farfield. The prism layer contained 32 layers and a $y^{+}$value of 1.0 computed from free stream conditions was used for the wall spacing. This process was performed for a single grid level only to later compare solver settings with the curvilinear simulations. This would help to ensure the solver settings were consistent in the LAVA framework and to also provide a third solution for comparison.

\section{Star-CCM+ Unstructured Grid Generation}

Three grid resolutions were generated with a coarse grid containing 17.2 million cells, medium grid of 49.7 million cells, and fine grid of 120 million cells shown in Fig. 7. For these meshes, the surface and volume meshes were generated using the polyhedral topology combined with the prism layer mesh of 31 layers. The total height of the prism layer was determined using the turbulent boundary layer thickness of the flat plate and the wall spacing for the coarse grid was again determined based on a $y^{+}$value of 1.0 computed from free stream conditions. Subsequent refinements for the medium and fine level meshes increase the number of points in the prism layer, but do not refine the $y^{+}$value. It was later determined that the Star-CCM+ grid generation and simulation procedure would be sufficient for database generation and thus LAVA Unstructured would only be used for the above mentioned purpose.

\section{Computational Results}

In LAVA, steady-state RANS simulations were used to perform a mesh refinement study using the curvilinear solver, while additional simulations using the unstructured solver were performed on a single grid level. Additional angle of attack $(\alpha)$ sweeps were also conducted using the curvilinear solver only. For both the curvilinear and unstructured simulations, a second-order convective flux discretization with van Albada slope limiter was determined to be the most appropriate numerical approach for this study. It was initially unknown whether the flow at this condition could be considered fully laminar, fully turbulent or contains transition, so both fully laminar and fully turbulent simulations were tested to determine the most probable flow regime. The SA turbulence model was therefore selected for simulations that were run with turbulence modeling enabled, while turbulence modeling was disabled for laminar simulations. Additionally, the effects of low Mach number preconditioning were also assessed due to the low subsonic, incompressible nature of these flow conditions [16].

For the Star-CCM+ simulations, the same mesh refinement study and angle of attack sweeps as LAVA Curvilinear were performed. In these simulations, the flow was resolved using the coupled compressible flow solver with low Mach number preconditioning. The inviscid fluxes were evaluated using the second order Weiss-Smith preconditioned Roe's flux differencing scheme with Venkatakrishnan flux limiter, and time-integration was performed using the Euler implicit scheme with linearized fluxes. The Spalart-Allmaras turbulence model was used with fully turbulent flow approximation to resolve the turbulence. Unless required due to asymmetric control surface deflection and flow, the half-body model using a symmetry plane was used for simulation, while LAVA utilized a full-body model for all simulations.

Intel Ivy Bridge E5-2680v2 nodes on the Pleiades supercomputer at NASA Ames Research Center were used to perform the LAVA curvilinear computation at 920 cores for 80 million grid points, 1,780 cores for 159 million grid points, and 3,580 cores for 322 million grid points, which translates into approximately 100,000 nodes per core. These simulations were run between 24 to 48 hours, unless the standard deviation in the drag coefficient fell below 1.0E-05. Star-CCM computations were performed on a cluster located at NASA Armstrong Flight Research Center. Although varying number of nodes and CPU types were used in the computations due to cluster availability, approximately 100,000 to 200,000 cells per core were utilized. Individual simulations typically required 24 to 48 hours to finish.

For the mesh refinement study explained in subsection V.A. all simulations were performed in free-air at the reference conditions discussed previously. Angle of attack sweep simulations described in subsection V.B use these same reference conditions and were performed in free-air and also enclosed within wind tunnel walls. Final database results in subsection V.C were again performed in free air at new reference conditions that will be discussed.

\section{A. Mesh Refinement Study}

The overset structured and unstructured grids previously discussed were used for the mesh refinement study, which consisted of coarse, medium and fine grids. This study was not only used to assess the grid dependence on the CFD solution, but also to determine solver settings that are most appropriate for this geometry and flow condition. The coarse level grid is comparatively inexpensive to perform these studies and was therefore used to test the numerical 
formulations and turbulence options within LAVA. The condition tested was at Mach 0.052, sea-level ambient, with a Reynolds number per meter, $R e_{l}=369,000 \mathrm{~m}^{-1}$, identical to the condition tested in the tunnel. Subsonic inflow/outflow boundary conditions were prescribed on all farfield boundaries with adiabatic no-slip wall boundary conditions assigned to all aircraft surfaces. Additionally, control surfaces were deflected to $\delta_{a}=10.0^{\circ}, \delta_{r}=15.0^{\circ}$, and $\delta_{s}=7.5^{\circ}$. Table 2 compares $c_{L}$ and $c_{D}$ between variations of solver settings and turbulence modeling. Comparing the results to the experiment suggested that modeling the flow turbulent with low Mach preconditioning enabled is most consistent with the test data. Furthermore, it was noted that the standard deviation in the steady-state aerodynamic loads is reduced by two orders of magnitude when SA is enabled, further suggesting the numerics are most compatible with a turbulent assumption.

Table 2 Comparison of solver setting impacts to aerodynamic loads

\begin{tabular}{l|cc|cc|}
\cline { 2 - 5 } & \multicolumn{2}{c|}{ LAVA Curvilinear } & \multicolumn{2}{c|}{ LAVA Unstructured } \\
\cline { 2 - 5 } & $c_{L}$ & $c_{D}$ & $c_{L}$ & $c_{D}$ \\
\hline Laminar without Preconditioning & 0.579 & 0.151 & 1.016 & 0.171 \\
Laminar with Preconditioning & 0.585 & 0.146 & 0.664 & 0.160 \\
SA Turbulence without Preconditioning & 1.015 & 0.119 & 1.112 & 0.154 \\
SA Turbulence with Preconditioning & 1.007 & 0.114 & 1.066 & 0.125 \\
\hline Experiment & 1.068 & 0.099 & 1.068 & 0.099 \\
\hline
\end{tabular}

Now that settings have been established for LAVA and Star-CCM+ for low-speed simulations, the appropriate mesh resolution must also be determined for database simulations. Coarse, medium and fine grid simulations were submitted for this same condition and the resulting aerodynamic loads are presented in Table 3. Visualizing the dimensionless pressure distribution, $c_{P}$, in Fig. 8 at various wing locations further illustrate how the field variables converge to a particular solution with mesh resolution.

All three grid solutions were deemed to be sufficiently close to the asymptotic convergence regime, with small change between medium and fine for both lift and drag. Figure 9 illustrates this trend by visualizing drag as a function of normalized effective grid spacing. Both codes predict a linear decrease in drag as the mesh approaches infinite resolution, or as $N^{-2 / 3}$ approaches zero, and both codes appear to approach nearly the same drag value at this infinite resolution. This asymptotically converged drag value for LAVA is about 0.1099 and for Star-CCM+ about 0.1111 , or a 12 count difference in drag. Relative to LAVA, the difference in the converged drag value is $1.1 \%$, which was determined satisfactory for this level of geometric complexity. Lift similarly converged to a value sufficiently close between the two codes, where the error relative to LAVA is $0.5 \%$ on the fine level. Because the resolution of the LAVA grids were all sufficiently fine to yield mesh independent results for lift, the fine grid lift values for both codes were used to obtain this error. It was decided that the medium refinement level was sufficiently fine to conduct all future simulations for both LAVA Curvilinear and StarCCM based on the mesh refinement results. Although the percent error for both codes relative to the experimental values is larger than the code to code variation, certain factors pertaining to the experimental set-up explain this discrepancy. The following sections will address alternative simulation strategies that can be used to close the gap between the uncorrected experimental data and the CFD predictions before database generation begins.

Table 3 Comparison of aerodynamic loads at simulated configurations

\begin{tabular}{l|cc|cc|}
\cline { 2 - 5 } Grid & \multicolumn{2}{|c|}{ LAVA Curvilinear } & \multicolumn{2}{c|}{ Star-CCM+ Unstructured } \\
\cline { 2 - 5 } & $c_{L}$ & $c_{D}$ & $c_{L}$ & $c_{D}$ \\
\hline Coarse & 1.0072 & 0.1138 & 1.0867 & 0.1274 \\
Medium & 1.0070 & 0.1121 & 1.0599 & 0.1198 \\
Fine & 1.0070 & 0.1115 & 1.0122 & 0.1152 \\
\hline Experiment & 1.068 & 0.099 & 1.068 & 0.099 \\
\hline
\end{tabular}




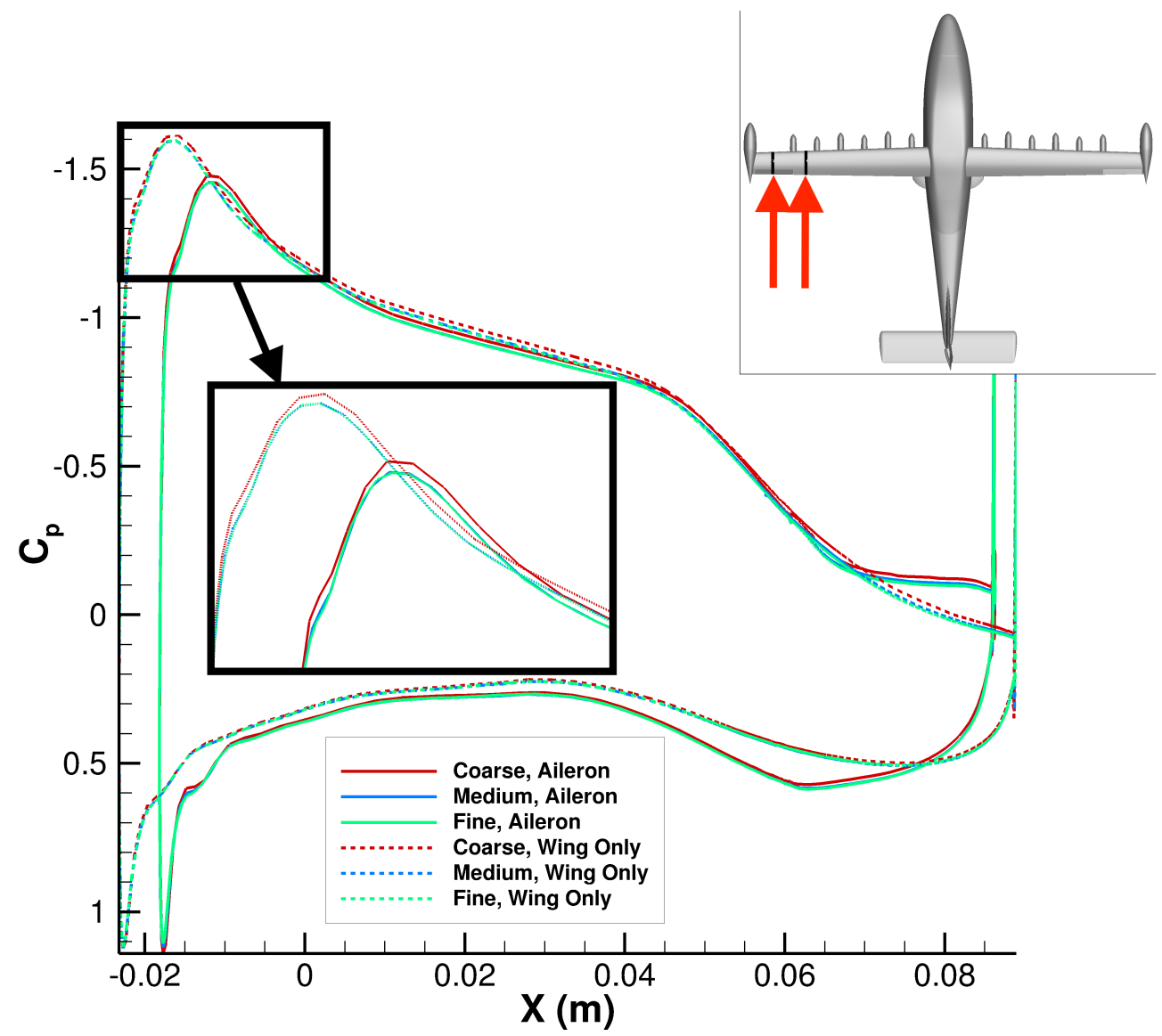

Fig. 8 LAVA Curvilinear pressure coefficient distributions for each grid level at clean wing location $(y=$ $\mathbf{- 0 . 6 5 8 m})$ and wing-aileron location $(y=-\mathbf{- 0 . 8 1 1 m})$.

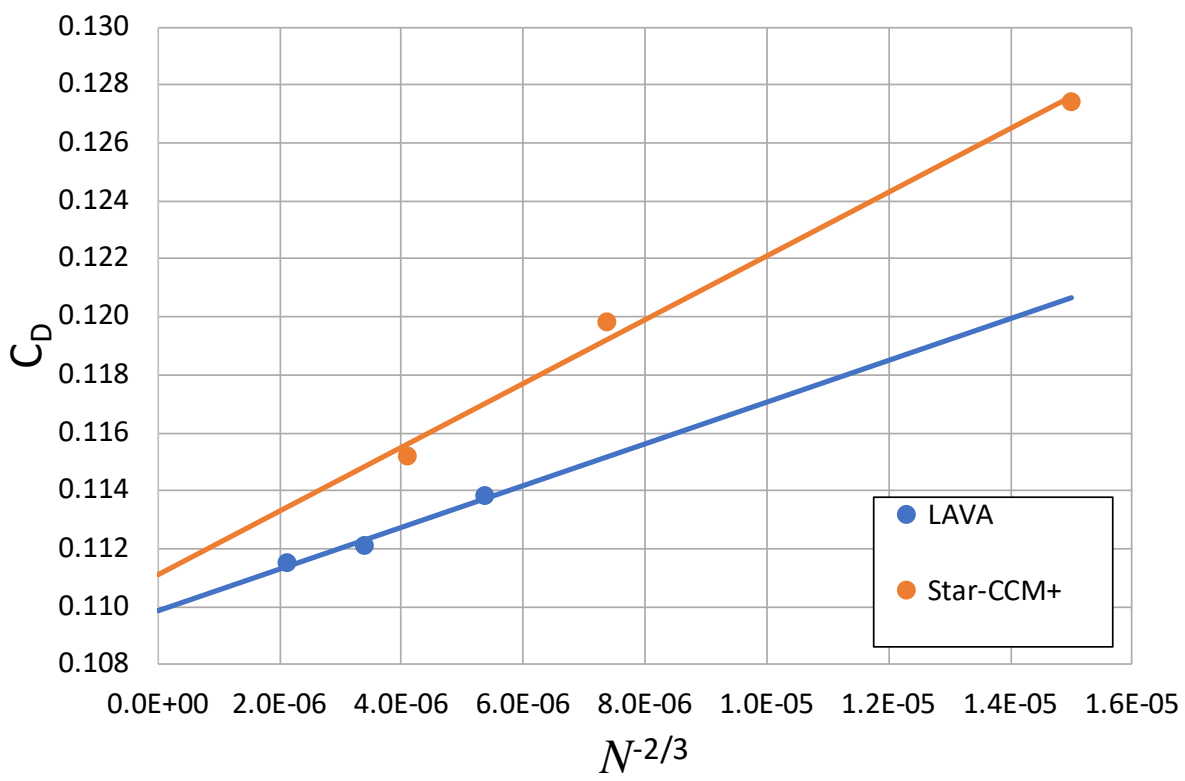

Fig. 9 Asymptotic convergence trends for the LAVA Curvilinear and Star-CCM+ mesh refinement study. 


\section{B. Wind Tunnel Effects and Angle of Attack Sweep}

Grid resolution and solver parameters have now been established through the mesh convergence study, additional cases varying aircraft configuration and flow condition were tested. It was assumed that the majority of the error between simulated values and experimental data (Table 3 ) was due to the blockage effects of the wind tunnel, phenomena that are not captured using free air CFD simulations. Although corrections are often made to wind tunnel data to account for blockage effects, this particular experimental data did not include such corrections. This would account for most of the CFD and experimental disagreement [17]. This is particularly true for low-speed applications, in which wind tunnel mounting hardware can cause flow disturbances that may easily propagate to the test article. A component build-up was therefore conducted to test this theory, where wind tunnel hardware was integrated into the overset grid system one component at a time. This approach would demonstrate which wind tunnel component, if any, would have the most significant impact on aerodynamic performance. First, the test article mounting apparatus known as the sting was integrated into the grid system. It was hypothesized that the wake of this structure would cause flow perturbations near the test article that would impact the pressure loading. Second, the stand which supports the sting and attaches to the wind tunnel floor was integrated along with the wind tunnel walls. These updates to the grid system are illustrated in Fig. 10. Geometry for the entire tunnel was not available for these simulations, therefore to replicate the 12-ft. NASA Langley Low-Speed Tunnel [18], a 12-ft. by 12ft. octagonal cross-section test section was constructed around the test article, and then extended 80 body lengths upstream and downstream. Boundary conditions assigned to the wind tunnel grids were adiabatic slip-walls and subsonic inflow/outflow at the wind tunnel entrance and exit.
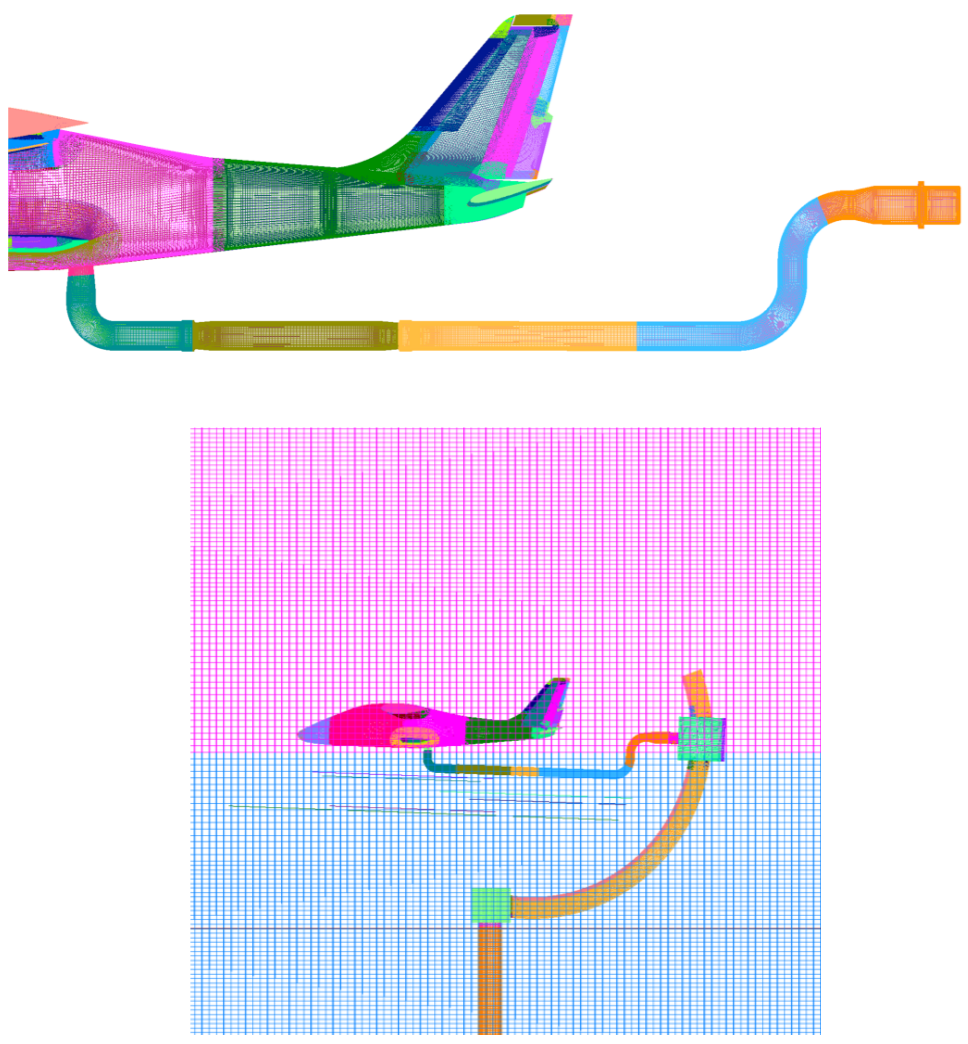

Fig. 10 Grid system for the $X-57$ test article plus sting (top) and test article plus sting, stand, and wind tunnel walls (bottom).

An aircraft configuration was selected for this build-up in which $\delta_{s}=-15^{\circ}$, all other controls were at their nominal settings, and the sting was rotated to produce a $2.0^{\circ}$ angle of attack. Results from this build-up are presented in Table 4 While both lift and drag are impacted by the addition of wind tunnel components, the error in lift for LAVA is reduced dramatically as this hardware is added; a $20.6 \%$ error in free air lift reduces to $17.8 \%$ with the sting addition and then $6.4 \%$ by adding the wind tunnel walls and sting. Visualizations of the flow field surrounding the test article and 
mounting system also indicate clear impacts as a result of the wind tunnel components. Figure 11 illustrates how the streamwise velocity component is significantly affected by the equipment at both the wing and aft body sections, confirming free air and wind tunnel fluid dynamics are substantially different, even if the same Reynolds and Mach numbers are simulated in both cases. Details to note include the vortex shedding behind the mounting fixture and the flow deceleration upstream of this apparatus, as well as increased flow acceleration over the wing location.

Table 4 Comparison of aerodynamic loads with and without modeling of wind tunnel hardware.

\begin{tabular}{l|ccccc|cccc|}
\cline { 2 - 9 } & \multicolumn{4}{c|}{ LAVA Curvilinear } & \multicolumn{4}{c|}{ Star-CCM+ Unstructured } \\
\cline { 2 - 9 } & $c_{L}$ & \% err. $c_{L}$ & $c_{D}$ & \% err. $c_{D}$ & $c_{L}$ & \% err. $c_{L}$ & $c_{D}$ & \% err. $c_{D}$ \\
\hline Free Air & 0.4575 & 20.6 & 0.0970 & 9.4 & 0.4691 & 18.6 & 0.1003 & 6.3 \\
Free Air + Sting & 0.4782 & 17.8 & 0.1003 & 6.3 & - & - & - & - \\
Wind Tunnel + Sting + Stand & 0.5394 & 6.4 & 0.0977 & 8.8 & 0.5307 & 7.9 & 0.0999 & 6.7 \\
\hline Experiment & 0.5762 & - & 0.1071 & - & 0.5762 & - & 0.1071 & - \\
\hline
\end{tabular}

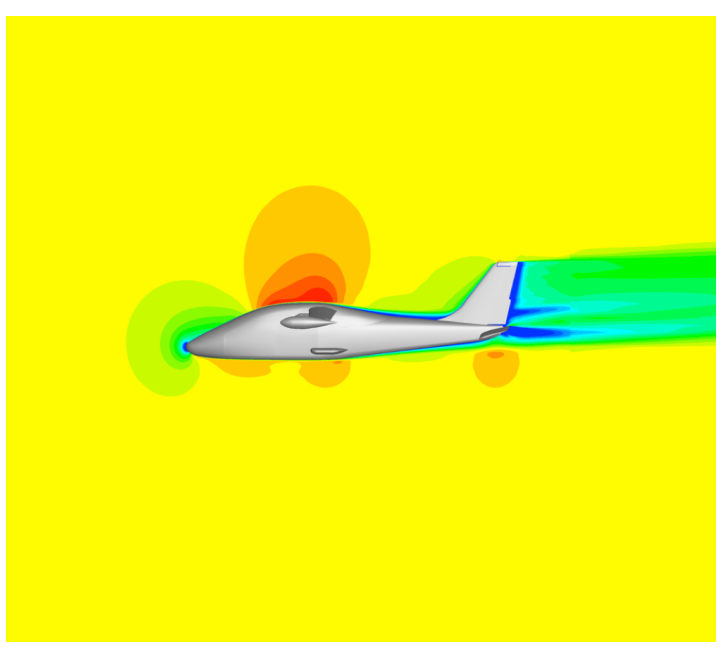

(a) Free Air

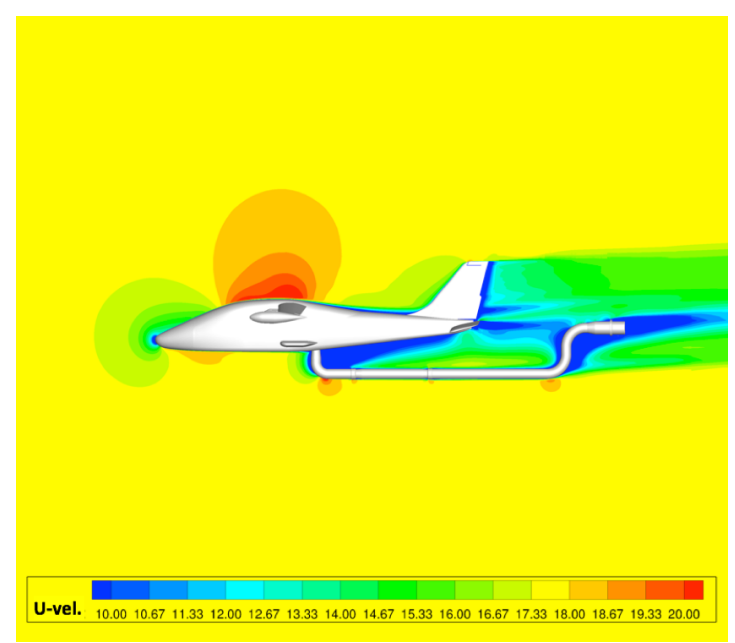

(b) Free Air + Sting

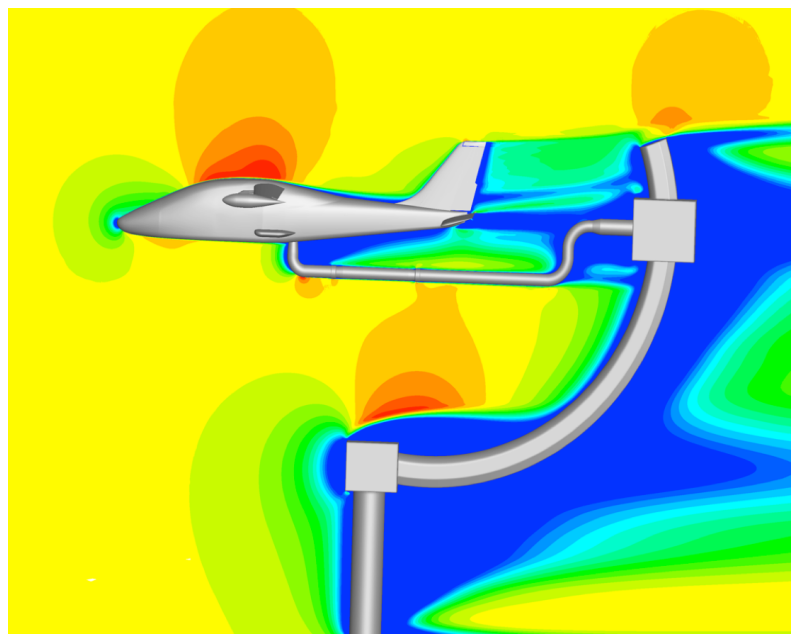

(c) Wind Tunnel + Sting + Stand

Fig. $11 U$-Velocity $(\mathrm{m} / \mathrm{s})$ contour for each wind tunnel build-up simulation. 
Due to the low subsonic free-stream Mach number of 0.052 , there is an apparent impact on the surface pressure loading as a result of upstream effects of the sting shown in Fig. 12 A lower pressure region near the wing leading edge is present when comparing the free air case to the free air + sting case, and this pressure difference becomes even more pronounced when comparing the free air + sting case with the wind tunnel + sting + stand case. This increased upper surface suction due to tunnel blockage interference is the main contributor to the increase in lift detailed in Table 4. Interestingly, not only do the sting and mounting fixture affect the pressure loading locally on the fuselage, but they also impact the loading on nonlocal components such as the wing as well. These findings support the idea that modeling all relevant test equipment with the test article is necessary when validating LAVA Curvilinear's predictions with the experiment, particularly when corrected data is unavailable.

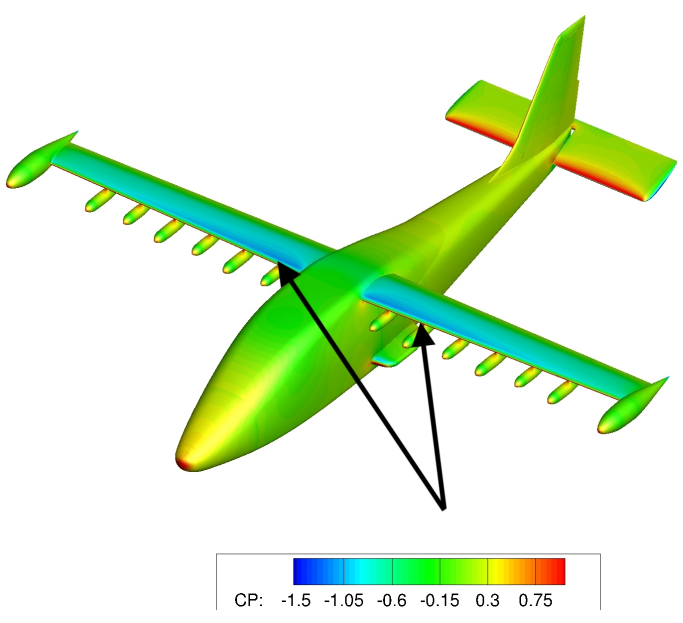

(a) Free Air

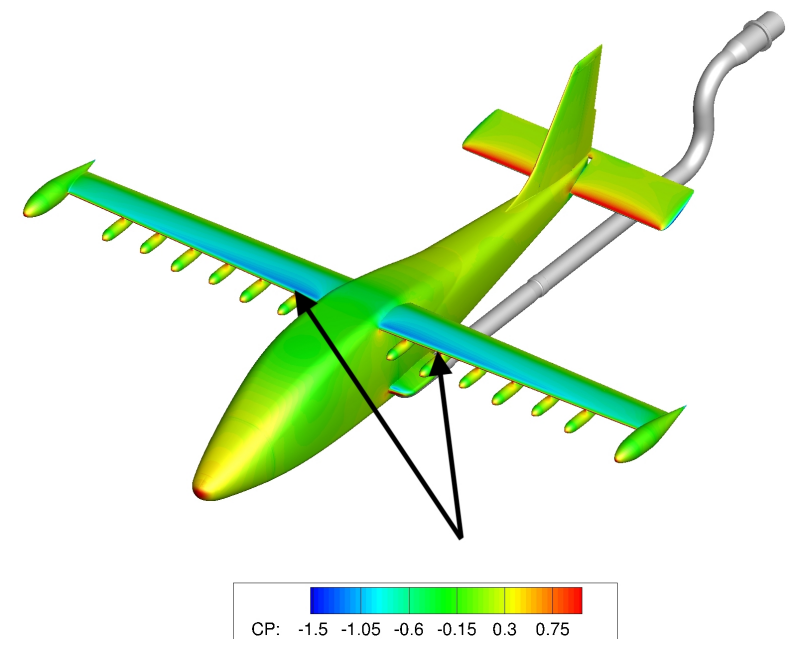

(b) Free Air + Sting

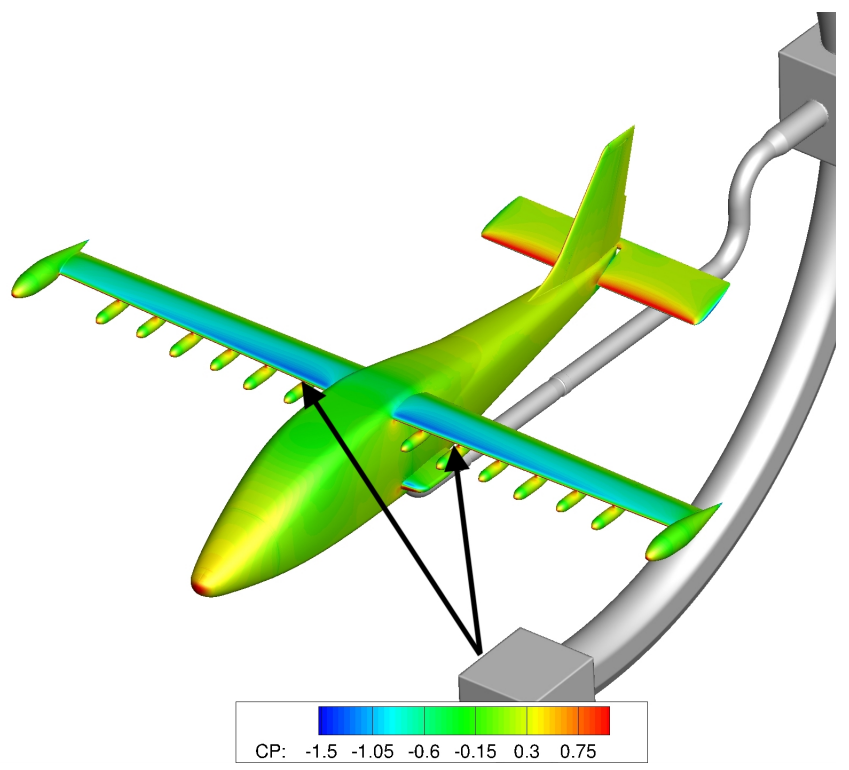

(c) Wind Tunnel + Sting + Stand

Fig. 12 Pressure coefficient contour for each wind tunnel build-up simulation. 
A closer look at the pressure distribution at a selected wing location further demonstrates how the component build-up impacts wing loading. A cut location between high-lift pylons 4 and $5(y=0.5497 \mathrm{~m})$ was selected to visualize these differences shown in Fig. 13 Due to the low-speed, essentially incompressible flow, the sting imparts a strong upstream flow field disturbance, slightly impacting the pressure loading on the wing as compared to the free air case. Similarly, when additional blockage is added by including the test article stand and wind tunnel walls, the wing loading is altered much more drastically. As tunnel components are added, progressively lower pressure is created on the wing upper surface while higher pressure is created on the wing lower surface, producing a net effect of increased lift as tunnel hardware is added to the simulation.

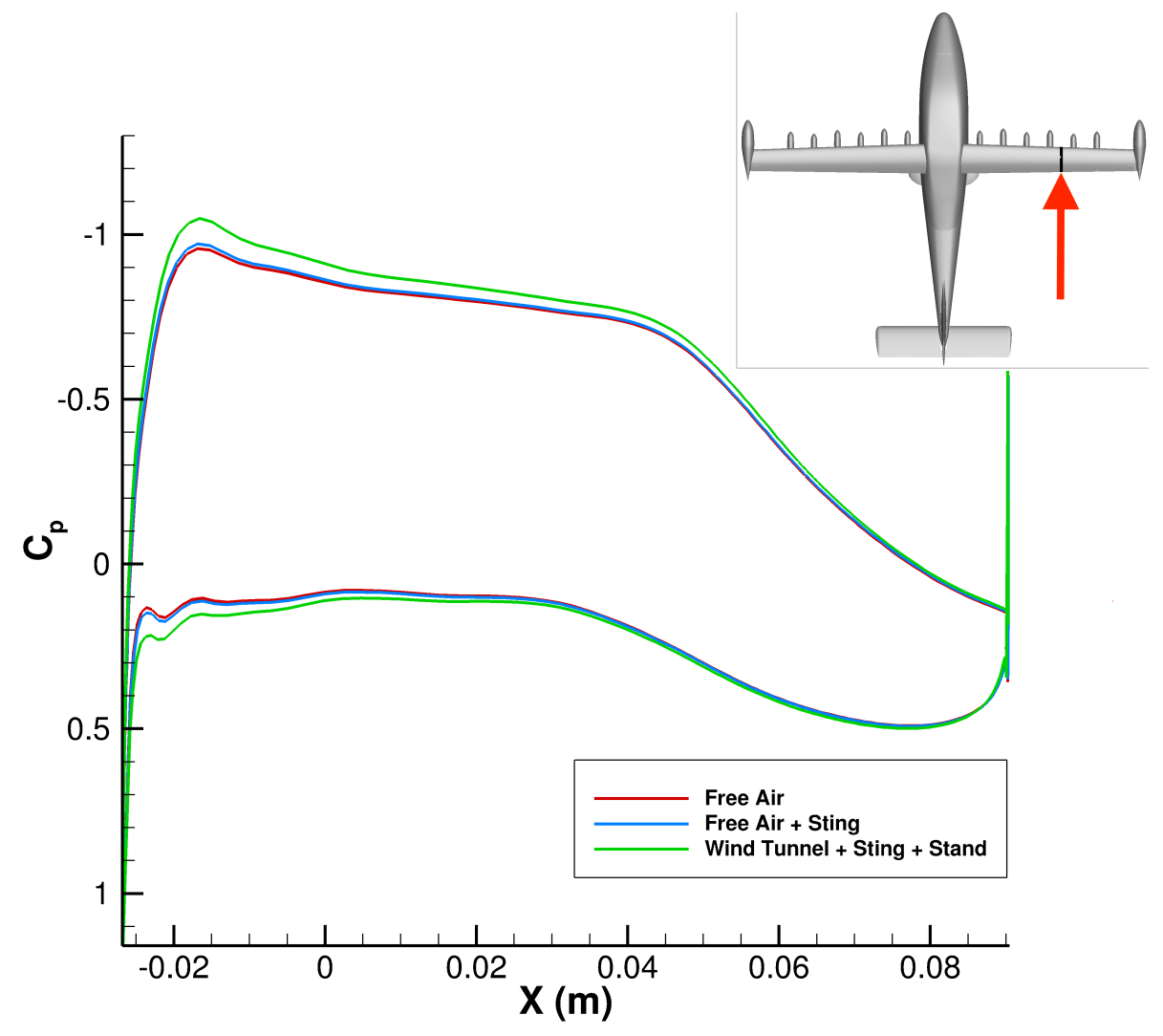

Fig. 13 Pressure coefficient on the wing for each component build-up simulation at spanwise location $y=$ $0.5497 \mathrm{~m}$.

The final pre-database study, an angle of attack sweep ranging from $0^{\circ}$ to $25^{\circ}$, presented in Fig. 14, compares the predicted loads for free air simulations and simulations that include wind tunnel hardware. The "Wall" denotes simulation with the tunnel wall and the sting with mounting device. The "WT" denotes the wind tunnel data. For the rest of the paper, "FS-simulation" and "Wall-simulation" will be used to denote simulation in free-stream and in wind tunnel, respectively. Both the FS- and Wall-simulation under predict the lift coefficient from $0.0^{\circ}$ to $25.0^{\circ}$ angle of attack. However, inclusion of the tunnel wall and its apparatus increases the lift coefficient and decreases the error. The drag coefficient compares well with the wind tunnel results up to $14.0^{\circ}$ angle of attack. For the angle of attack higher than $15.0^{\circ}$, simulation results under predict drag. Similarly for the lift coefficient, the addition of the wind tunnel apparatus in the model improves the comparison. The pitching moment for both the FS- and Wall-simulations are consistent up to $10.0^{\circ}$ angle of attack. However, at $17.0^{\circ}$ the FS-simulation dips to under predict whereas the Wall-simulation over predicts the pitching moment.

It should be reiterated that in many instances, corrected experimental data is provided in lieu of uncorrected data measured directly from a force balance. This will account and adjust for the effects of buoyancy, wall interference, stand blockage, and other extraneous effects on the test article which are not relevant in free-air flight and certain wind tunnel tests [19]. In these situations, a component build-up in the CFD may or may not be desired for a validation 
against experimental data should the corrected data be deemed accurate. In other situations, the raw uncorrected data may be assumed accurate enough for comparison with FS-simulation, which is not the case for this study. In the case of this X-57 study, uncorrected force balance measurements are the only data available and do not agree well with FS-simulations, necessitating the component build-up presented here for a valid comparison. Overall, results of this study are encouraging and suggest that LAVA and Star-CCM+ predict aerodynamic loads with decent accuracy, indicating database work may begin.

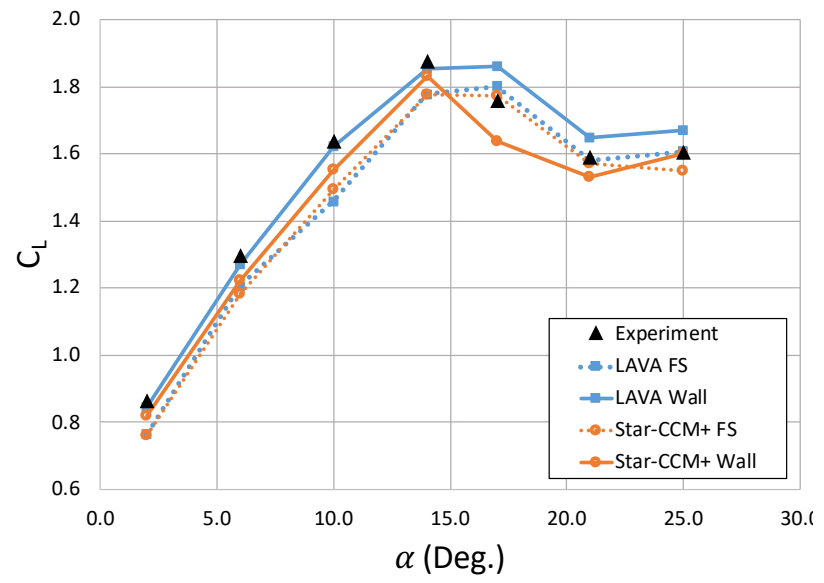

(a) Lift Coefficient

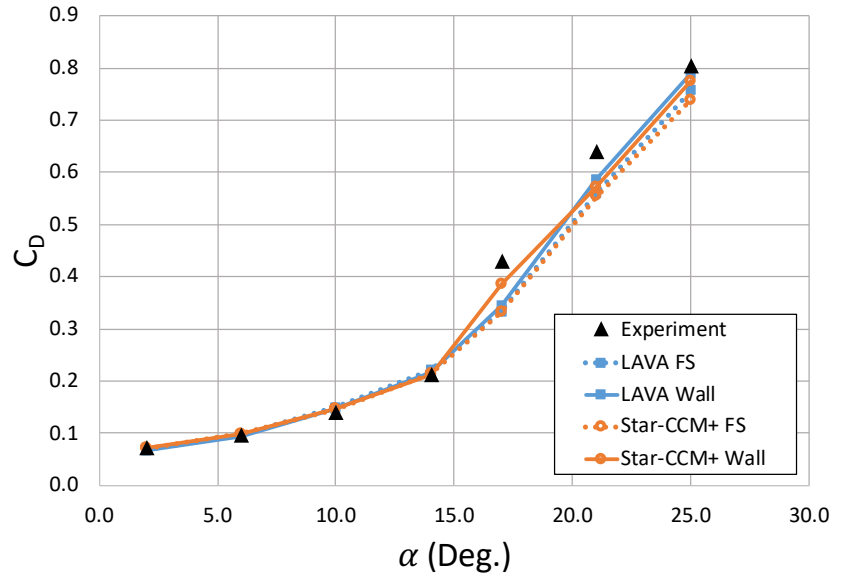

(b) Drag Coefficient

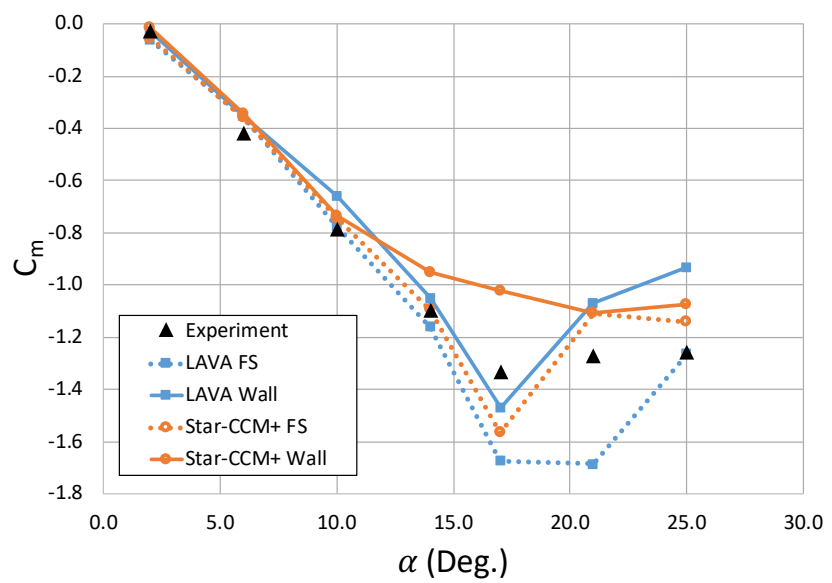

(c) Pitching Moment Coefficient

Fig. 14 Aerodynamic forces and pitching moments as a function of angle of attack.

\section{Applying Lessons Learned to an Aerodynamic Database}

The best practices established through the validation cases were then applied towards the first Mod. III database, which contained 216 power-off cases for a variety of configurations at different flap settings. Since a procedure had been developed to automatically deflect control surfaces to any desired angle, overset grid generation was a relatively quick procedure for most components. The structured overset grids used for the medium level during the initial refinement study could be reused since the geometry remained identical, with the exception of the flaps, high-lift pylons and a newly added vortex generator. Generation of flap grids required additional overhead and grid points in order to resolve geometrically complex features such as the flap cove and pylon/flap intersection. Fig 15 illustrates the three flap settings, nominal, medium, and maximum, that correspond to take-off, cruise and landing conditions respectively. A $32 \%$ increase in grid points from the nominal flap setting is required to resolve a mid-way deflected flap, while a $25 \%$ increase 
in grid points is required for the maximum flap setting. The change in total grid points as a result of all other control surface deflections is negligible.

A more comprehensive presentation of all database results will be given in future papers upon completion of the database, but preliminary results will be presented here for selected cases. For the nominal cruise flap setting, an initial angle of attack sweep was simulated with all control surfaces kept at neutral for a representative cruise condition, with Mach $=0.233$, altitude is $8,000 \mathrm{ft}$., and Reynolds number per meter, $R e_{l}$, is 4,330,000 $\mathrm{m}^{-1}$. All solver settings and reference dimensions outlined previously as a result of the validation cases were used for these simulations, with the exception of low Mach preconditioning since the Mach number increased. Angles of attack were selected based on the anticipated stall angle of attack, where more resolution would be given in the stall regime of the lift curve while less resolution would be given to the linear regime. Based on historical data for the GAW-1 airfoil, the cross-sectional shape of the X-57 wing, stall for this particular Reynolds number was measured at $16^{\circ}$ angle of attack [20]. Angles of attack to simulate were then selected with a resolution of $4^{\circ}$ pre-stall, $1^{\circ}$ around stall, and $2^{\circ}$ after stall. Although it was expected that the stall angle of attack would shift by incorporating other aircraft components, particularly the high-lift pylons, the results obtained by Star-CCM+ and LAVA Curvilinear predict a stall angle of attack very close to this experimental value. The loads computed for LAVA Curvilinear and Star-CCM+ are in very strong agreement with each other, even near stall as shown in Fig. 16. In the pre-stall linear regime for lift, LAVA Curvilinear's predictions are within $0.5 \%$ of Star-CCM+ while post-stall a difference of less than $8 \%$ is observed. For both drag and pitching moment, LAVA Curvilinear predicts within 5\% of Star-CCM+ for both pre-stall and post-stall.

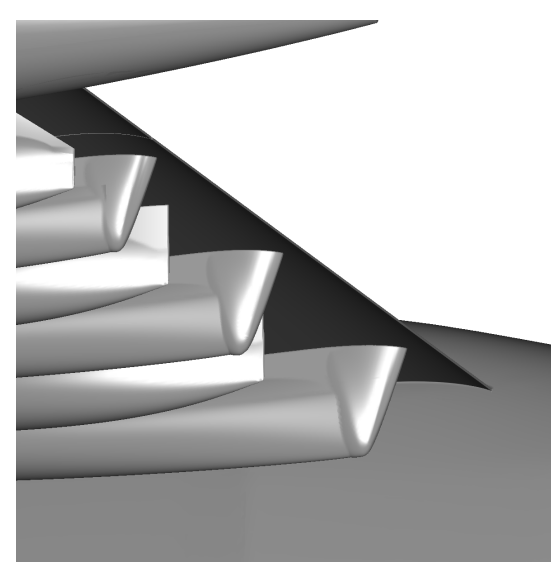

(a) Flaps $0^{\circ}$, Cruise Setting, 123.9 million points

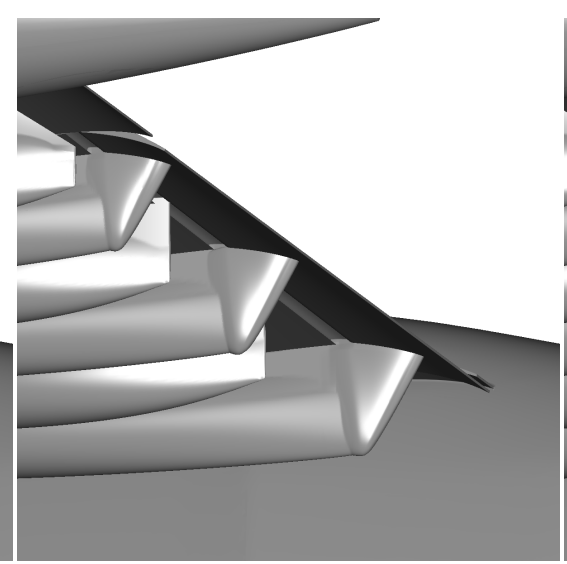

(b) Flaps $10^{\circ}$, Take-off Setting, 163.9 million points

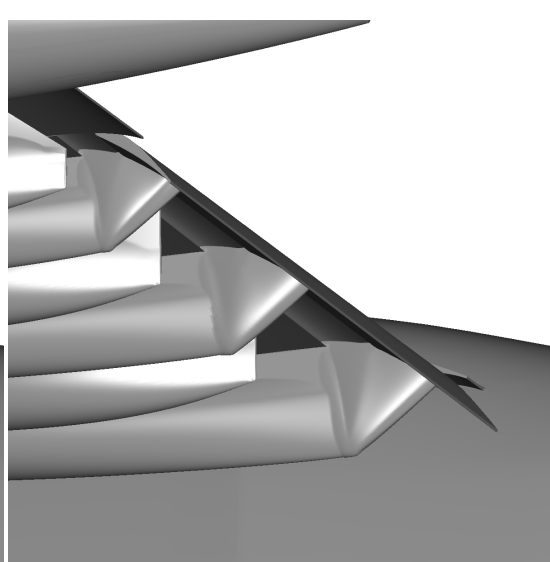

(c) Flaps $30^{\circ}$, Landing Setting, 154.4 million points

Fig. 15 Overset mesh surfaces for each flap setting.

A closer analysis of the pressure distribution and streamlines confirms that the agreement in integrated quantities is indeed a result of flow field similarities and not due to a possible cancellation in loading errors. Figure [17]compares the flow structures via pressure distribution and surface streamlines at selected angles of attack between the two codes pre-stall, at stall, and post-stall. For the $4^{\circ}$ pre-stall case, laminar flow dominates the wing upper surface on both codes with a similar pressure distribution present for each. This behavior is also observed for higher pre-stall angles of attack with vortical structures beginning to form due to the wake of the high lift pylons. These structures become much more pronounced as the stall angle of attack of $16^{\circ}$ is reached, where separated flow is seen aft of the half chord location behind the high-lift pylons and further outboard at the aileron location. Finally, at $22^{\circ}$ angle of attack, near total flow separation is predicted for the entirety of the wing, with pockets of attachment still between various high lift pylons.

Strong code-to-code agreement is observed for both the integrated quantities and the flow fields for the entire alpha sweep despite the tendency for steady-state RANS to bifurcate in regions of separated flow. Indeed the differences in CFD solutions become more pronounced post-stall, but the relative error between the codes is well within acceptable bounds as compared to the High Lift Prediction Workshop scatter in the stall regime [21]. These results are especially encouraging due to the fact that entirely different mesh paradigms and CFD solvers have independently predicted pre-stall and post-stall flow characteristics. Furthermore, this demonstrated taking time prior to database generation to establish best practices for turbulence modeling, numerical scheme, grid resolution, etc. was a worth-while investment since only one simulation attempt for each code was required for this agreement. The remainder of the database 
simulations could now begin with minimal concern regarding solution accuracy. It is anticipated that spot-checks for selected cases will be made in which the flow field would be visualized and compared between the codes to ensure consistency. However, current results indicate that simulations can confidently be run in parallel with little concern for wasted compute resources. Results of this power-off database and future power-on databases will be reported in future AIAA publications upon completion.

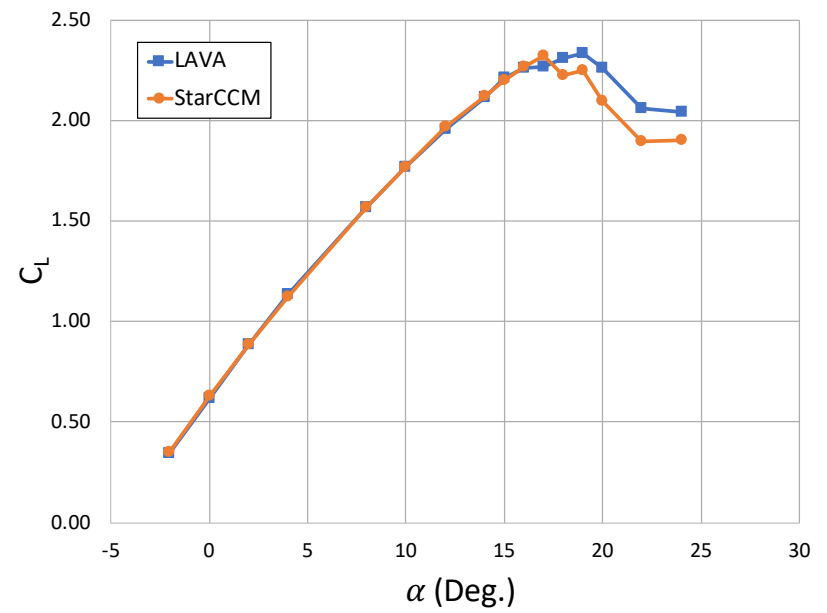

(a) Lift Coefficient

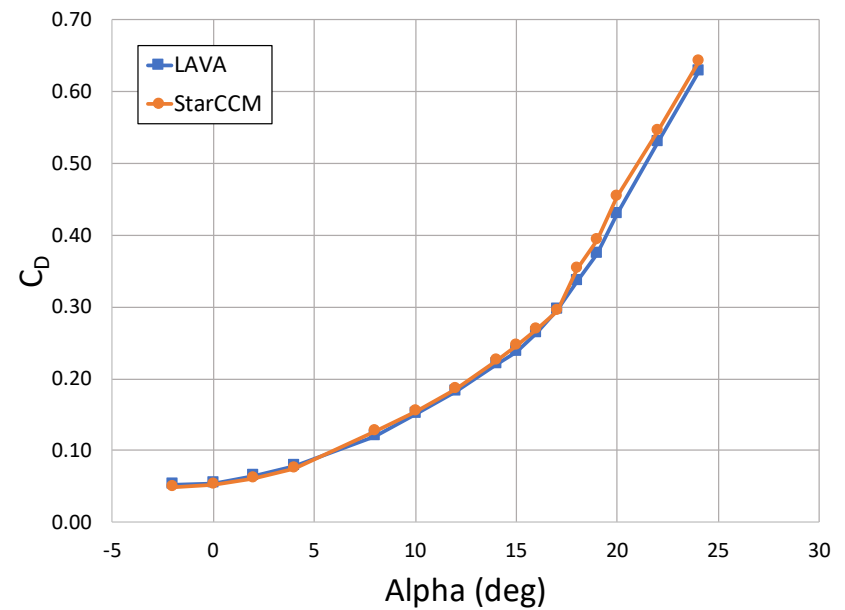

(b) Drag Coefficient

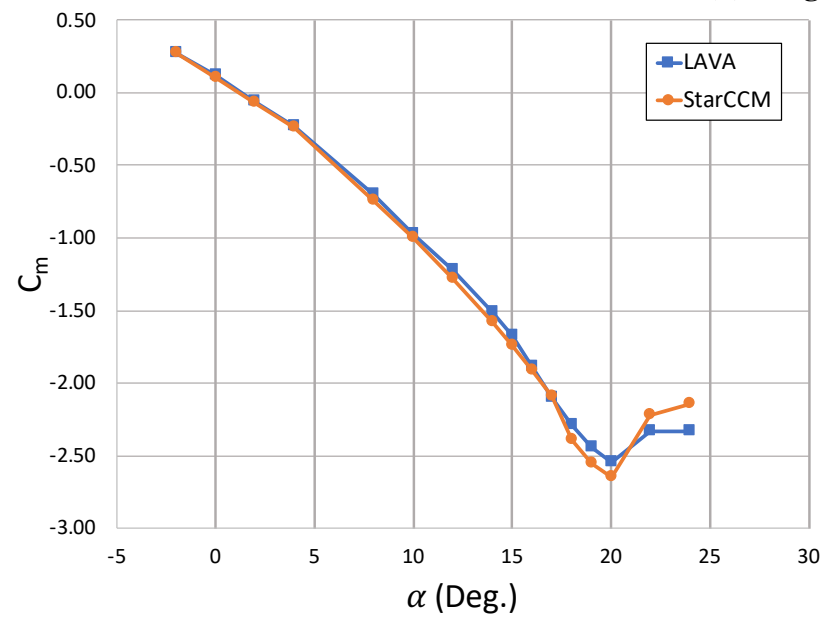

(c) Pitching Moment Coefficient

Fig. 16 Aerodynamic forces and pitching moments as a function of angle of attack for nominal flap setting.

\section{Conclusions}

The intent of this study is to establish best practices prior to database generation, including techniques for efficient mesh generation, ways to determine appropriate solver settings, and methodologies to validate against experimental data for geometries like the X-57. Consequences of this up-front effort result in efficient database generation with optimal accuracy for each simulated case. Results from this preliminary work have also emphasized the importance of mesh resolution studies and code-to-code comparison before extensive resources are used for the database. It was through this work that average error between experimental and simulated lift and drag was significantly reduced from above $20 \%$ to less than $5 \%$ using the proper simulation techniques.

Transferring these lessons learned is crucial to a successful database generation, as demonstrated by preliminary database results. For example, developing a routine to automatically regenerate structured overset grids for changes in geometry reduced a turn around time on the order of days of human effort to a matter of minutes. This streamlined the 
pre-processing steps required for the multiple configurations that would be simulated. Furthermore, knowing the proper solver settings to use through CFD validation ensured that both LAVA Curvilinear and Star-CCM+could arrive at consistent, accurate solutions on a first attempt. This was proven by both codes' ability to predict the same stall angle of attack and $C_{L, \max }$ within $0.6 \%$, which is an exceptional accomplishment for steady-state RANS. Further analysis of flow features on the wing across the angle of attack sweep indicate both codes are capable of achieving consistent solutions, despite entirely different mesh paradigms and solver algorithms. The validation simulations and initial database alpha sweep ensure that the accuracy of any future database simulations is maximized and scrutinization of every individual case, unless deemed suspicious, is no longer necessary.

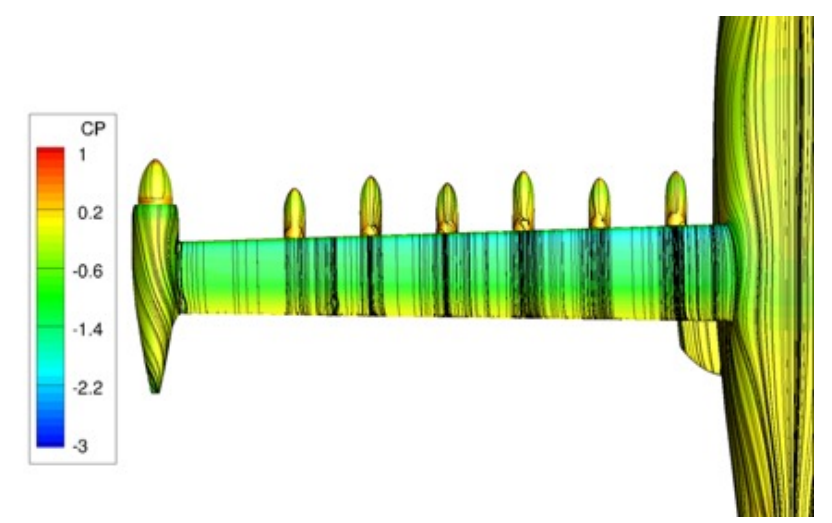

(a) $4^{\circ}$ angle of attack, LAVA Curvilinear

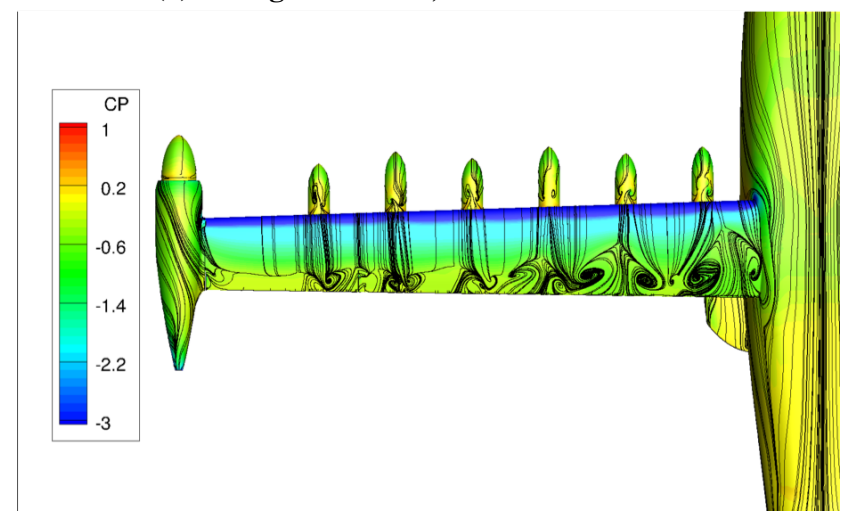

(c) $16^{\circ}$ angle of attack, LAVA Curvilinear

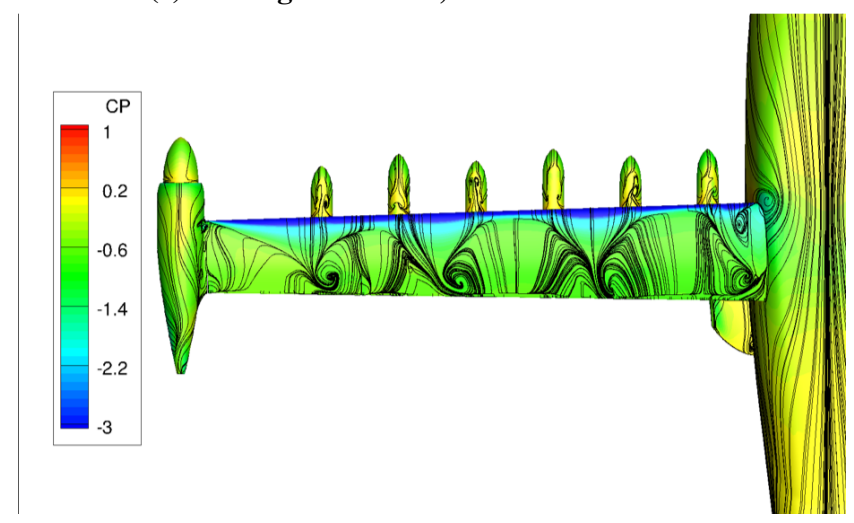

(e) $22^{\circ}$ angle of attack, LAVA Curvilinear

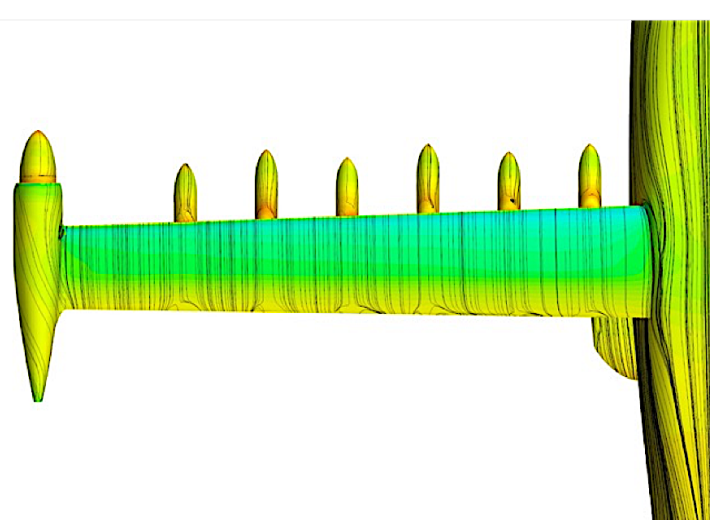

(b) $4^{\circ}$ angle of attack, Star-CCM+

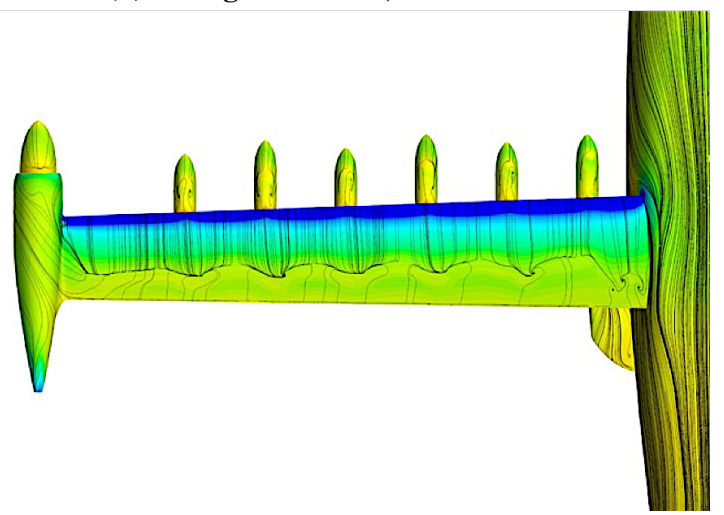

(d) $16^{\circ}$ angle of attack, Star-CCM+

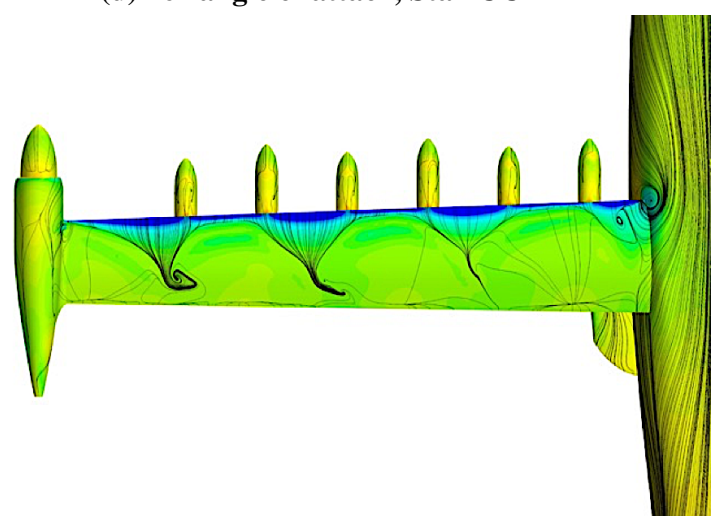

(f) $22^{\circ}$ angle of attack, Star-CCM+

Fig. 17 Surface streamlines and pressure distributions for selected pre-stall, stall and post-stall conditions. Note: Particle seeding for streamlines randomly distributed for each subfigure. 


\section{Acknowledgments}

This project was funded by the Scalable Convergent Electric Propulsion Technology and Operations Research (SCEPTOR) sub-project in the Flight Demonstrations and Capabilities (FDC) project, which is under the Integrated Aviation Systems Program (IASP) in the NASA Aeronautics Research Mission Directorate (ARMD). The authors appreciate the support given by the NASA Armstrong Aerodynamics and Propulsion Research Branch and David Cox at NASA Langley Research Center for providing the experimental data. The authors also appreciate the support and technical insight given by Michael Frederick and Trong Bui of NASA Armstrong and Jeffrey Viken of NASA Langley over the duration of this study. Results, grids, and knowledge shared between these individuals and groups have been integral in conducting a successful analysis. Computer resources were provided by the NASA Advanced Supercomputing (NAS) Pleiades facility.

\section{References}

[1] "NASA Armstrong Fact Sheet: NASA X-57 Maxwell," 2017. https://www.nasa.gov/centers/armstrong/news/ FactSheets/FS-109.html

[2] Kiris, C. C., Housman, J. A., Barad, M. F., Brehm, C., Sozer, E., and Moini-Yekta, S., "Computational framework for launch, ascent, and vehicle aerodynamics (LAVA)," Aerospace Science and Technology, Vol. 55, 2016, pp. 189-219.

[3] Siemens, "Simcenter Star-CCM+," 2018. www.cd-adapco.com/products/star-ccm.

[4] Beutel, A., "NASA Electric Research Plane Gets X Number, New Name," 2016. https://www.nasa.gov/press-release/ nasa-electric-research-plane-gets-X-number-new-name

[5] Cole, S., "12 Foot Low-Speed Tunnel (12 FT LST)," 2018. https://researchdirectorate.larc.nasa.gov/12-footlow-speed-tunnel-12-ft-lst/.

[6] Spalart, P., and Allmaras, S., "A one-equation turbulence model for aerodynamic flows," Proceedings of the 30th aerospace sciences meeting and exhibit, 1992, p. 439.

[7] Liou, M.-S., “A sequel to ausm: Ausm+," Journal of computational Physics, Vol. 129, No. 2, 1996, pp. 364-382.

[8] Liou, M.-S., "Ten years in the making-AUSM-family," 15th AIAA Computational Fluid Dynamics Conference, 2001 , p. 2521.

[9] Venkatakrishnan, V., "On the convergence of limiters and convergence to steady state solutions," Tech. rep., AIAA-93-0880, 1994.

[10] BETA-CAE, "ANSA Pre-processor, The Advanced CAE Pre-processing Software for Complete Model Build-up," 2018. http://www.beta-cae.com/ansa.htm

[11] Pointwise, "Software and Services for CFD Mesh Generation," 2018. http://www . pointwise.com/products/index.html

[12] “3rd AIAA CFD High Lift Prediction Workshop Gridding Guidelines,” 2016, pp. 1-2. https://hiliftpw. larc.nasa. gov/Workshop3/GriddingGuidelines-HiLiftPW3-v10.pdf

[13] Chan, W. M., "Overset grid technology development at NASA Ames Research Center," Computers \& Fluids, Vol. 38, No. 3, 2009, pp. 496-503.

[14] Chan, W. M., and Buning, P. G., "Surface grid generation methods for overset grids," Computers \& fluids, Vol. 24, No. 5, 1995, pp. 509-522.

[15] Joy, K. I., “Quadratic Bezier curves,” Department of Computer Science, University of California,, Davis, 2000, pp. 1-6.

[16] Turkel, E., "Preconditioned methods for solving the incompressible and low speed compressible equations," Journal of computational physics, Vol. 72, No. 2, 1987, pp. 277-298.

[17] Maskell, E., "A theory of the blockage effects on bluff bodies and stalled wings in a closed wind tunnel," Tech. rep., AERONAUTICAL RESEARCH COUNCIL LONDON (UNITED KINGDOM), 1963.

[18] Ball, W., “12-Foot Low Speed Tunnel,” Tech. rep., 2016. https://crgis.ndc.nasa.gov/historic/12-Foot_Low_ Speed_Tunnel

[19] Whipple, R. D., and Ricket, J. L., "Low-speed aerodynamic characteristics of a 1/8-scale X-29A airplane model at high angles of attack and sideslip, NASA-TM-87722," 1986. 
[20] Mcghee, R., and Beasley, W., "Low speed aerodynamic characteristics of a 17 percent thick airfoil section designed for general aviation applications, NASA-TM-X-72697,” 1973.

[21] Rumsey, C. L., Slotnick, J. P., and Sclafani, A. J., "Overview and Summary of the Third AIAA High Lift Prediction Workshop," 2018 AIAA Aerospace Sciences Meeting, 2018, p. 1258. 